\title{
PRIMORDIAL NON-GAUSSIANITY, SCALE-DEPENDENT BIAS, AND THE BISPECTRUM OF GALAXIES
}

\author{
Donghui JeOng AND EIICHIRo Komatsu \\ Texas Cosmology Center, University of Texas at Austin, 1 University Station, C1400, Austin, TX 78712, USA; djeong @ astro.as.utexas.edu \\ Received 2009 May 20; accepted 2009 August 10; published 2009 September 8
}

\begin{abstract}
The three-point correlation function of cosmological fluctuations is a sensitive probe of the physics of inflation. We calculate the bispectrum, $B_{g}\left(k_{1}, k_{2}, k_{3}\right)$, Fourier transform of the three-point function of density peaks (e.g., galaxies), using two different methods: the Matarrese-Lucchin-Bonometto formula and the locality of galaxy bias. The bispectrum of peaks is not only sensitive to that of the underlying matter density fluctuations, but also to the four-point function. For a physically motivated, local form of primordial non-Gaussianity in the curvature perturbation, $\Phi=\phi+f_{\mathrm{NL}} \phi^{2}+g_{\mathrm{NL}} \phi^{3}$, where $\phi$ is a Gaussian field, we show that the galaxy bispectrum contains five physically distinct pieces: (1) non-linear gravitational evolution, (2) non-linear galaxy bias, (3) $f_{\mathrm{NL}},(4) f_{\mathrm{NL}}^{2}$, and (5) $g_{\mathrm{NL}}$. While (1), (2), and a part of (3) have been derived in the literature, (4) and (5) are derived in this paper for the first time. We also find that, in the high-density peak limit, (3) receives an enhancement of a factor of $\sim 15$ relative to the previous calculation for the squeezed triangles $\left(k_{1} \approx k_{2} \gg k_{3}\right)$. Our finding suggests that the galaxy bispectrum is more sensitive to $f_{\mathrm{NL}}$ than previously recognized, and is also sensitive to a new term, $g_{\mathrm{NL}}$. For a more general form of local-type non-Gaussianity, the coefficient $f_{\mathrm{NL}}^{2}$ can be interpreted as $\tau_{\mathrm{NL}}$, which allows us to test multi-field inflation models using the relation between the three- and four-point functions. The usual terms from Gaussian initial conditions, (1) and (2), have the smallest signals in the squeezed configurations, while the others have the largest signals; thus, we can distinguish them easily. We cannot interpret the effects of $f_{\mathrm{NL}}$ on $B_{g}\left(k_{1}, k_{2}, k_{3}\right)$ as a scale-dependent bias, and thus replacing the linear bias in the galaxy bispectrum with the scaledependent bias known for the power spectrum results in an incorrect prediction. As the importance of primordial non-Gaussianity relative to the non-linear gravity evolution and galaxy bias increases toward higher redshifts, galaxy surveys probing a high-redshift universe are particularly useful for probing the primordial non-Gaussianity.
\end{abstract}

Key words: cosmology: theory - large-scale structure of universe

Online-only material: color figures

\section{INTRODUCTION}

Are primordial fluctuations Gaussian or non-Gaussian? The simplest models of inflation, driven by a slowly rolling single scalar field with the canonical kinetic term originated from the Bunch-Davis vacuum, predict the amplitude of primordial non-Gaussianity that is below the detectable level. Therefore, a convincing detection of primordial non-Gaussianity would rule out the above simplest models, and thus lead to a breakthrough in our understanding of the physics of inflation (see Bartolo et al. 2004, for a review).

The tightest limit on primordial non-Gaussianity so far comes from the angular bispectrum, spherical harmonic transform of the angular three-point correlation function (see Komatsu 2001, for a review), of anisotropy in the cosmic microwave background (CMB) radiation (see Komatsu et al. 2009; Smith et al. 2009; Curto et al. 2009, for the latest limits).

The large-scale structure of the universe can also provide alternative ways of probing primordial non-Gaussianity through abundances and clustering properties of galaxies and clusters of galaxies. However, as the large-scale structure of the universe is more non-linear than $\mathrm{CMB}$, it was generally thought that $\mathrm{CMB}$ would be the most promising way of constraining primordial non-Gaussianity (Verde et al. 2000).

On the other hand, Sefusatti \& Komatsu (2007) have shown that observations of the large-scale structure of the universe in a high-redshift universe, i.e., $z>1$, can provide competitive limits on primordial non-Gaussianity, as the other non-linear effects are weaker in a high-redshift universe. Specifically, they calculate the bispectrum of the three-dimensional distribution of galaxies, $B_{g}\left(k_{1}, k_{2}, k_{3}\right){ }^{1}$ on large scales as (see also Scoccimarro et al. 2004)

$$
\begin{aligned}
& B_{g}\left(k_{1}, k_{2}, k_{3}, z\right)=3 b_{1}^{3} f_{\mathrm{NL}} \Omega_{m} H_{0}^{2} \\
& \quad \times\left[\frac{P_{m}\left(k_{1}, z\right)}{k_{1}^{2} T\left(k_{1}\right)} \frac{P_{m}\left(k_{2}, z\right)}{k_{2}^{2} T\left(k_{2}\right)} \frac{k_{3}^{2} T\left(k_{3}\right)}{D(z)}+(2 \text { cyclic })\right] \\
& \quad+2 b_{1}^{3}\left[F_{2}^{(s)}\left(\boldsymbol{k}_{1}, \boldsymbol{k}_{2}\right) P_{m}\left(k_{1}, z\right) P_{m}\left(k_{2}, z\right)+(2 \text { cyclic })\right] \\
& +b_{1}^{2} b_{2}\left[P_{m}\left(k_{1}, z\right) P_{m}\left(k_{2}, z\right)+(2 \text { cyclic })\right],
\end{aligned}
$$

where $H_{0}$ and $\Omega_{m}$ are the present-day value of Hubble's constant and the matter density parameter, respectively, $P_{m}(k, z)$ is the power spectrum of linear matter density fluctuations, $D(z)$ is the linear growth factor, $T(k)$ is the linear transfer function whose limit is $T(k) \rightarrow 1$ as $k \rightarrow 0$, and $F_{2}^{(s)}\left(\boldsymbol{k}_{1}, \boldsymbol{k}_{2}\right)$ is a known mathematical function given by Bernardeau et al. (2002)

$$
F_{2}^{(s)}\left(\boldsymbol{k}_{1}, \boldsymbol{k}_{2}\right)=\frac{5}{7}+\frac{\boldsymbol{k}_{1} \cdot \boldsymbol{k}_{2}}{2 k_{1} k_{2}}\left(\frac{k_{1}}{k_{2}}+\frac{k_{2}}{k_{1}}\right)+\frac{2}{7}\left(\frac{\boldsymbol{k}_{1} \cdot \boldsymbol{k}_{2}}{k_{1} k_{2}}\right)^{2} .
$$

This function vanishes in the squeezed limit, $\boldsymbol{k}_{1}=-\boldsymbol{k}_{2}$ (the triangles with the maximum angle, i.e., $\pi$, between $\boldsymbol{k}_{1}$ and $\boldsymbol{k}_{2}$, and $\left.\left|\boldsymbol{k}_{1}\right|=\left|\boldsymbol{k}_{2}\right|\right)$, and takes on the maximum value, $F_{2}^{(s)}=(\alpha+1)^{2} /(2 \alpha) \geqslant 2$, in the opposite limit, $\boldsymbol{k}_{1}=\alpha \boldsymbol{k}_{2}$ where $\alpha \geqslant 1$ (the triangles with the vanishing angle between $\boldsymbol{k}_{1}$ and $\boldsymbol{k}_{2}$ ).

Here, $b_{1}$ and $b_{2}$ are the linear and non-linear galaxy bias parameters, respectively, which relate the underlying matter

\footnotetext{
1 The bispectrum, the Fourier transform of the three-point correlation function, is defined as $\left\langle\delta\left(\boldsymbol{k}_{1}\right) \delta\left(\boldsymbol{k}_{2}\right) \delta\left(\boldsymbol{k}_{3}\right)\right\rangle \equiv(2 \pi)^{3} B\left(k_{1}, k_{2}, k_{3}\right) \delta^{D}\left(\boldsymbol{k}_{1}+\boldsymbol{k}_{2}+\boldsymbol{k}_{3}\right)$.
} 
density contrast, $\delta_{m}$, to the galaxy density contrast, $\delta_{g}$, as (Fry \& Gaztanaga 1993)

$$
\delta_{g}(\boldsymbol{x})=b_{1} \delta_{m}(\boldsymbol{x})+\frac{b_{2}}{2}\left[\delta_{m}^{2}(\boldsymbol{x})-\sigma^{2}\right]+\cdots,
$$

where $\sigma^{2} \equiv\left\langle\delta_{m}^{2}\right\rangle$, which ensures $\left\langle\delta_{g}\right\rangle=0$.

The last two terms in Equation (1) are the well known results for Gaussian initial conditions (see Bernardeau et al. 2002, for a review), whereas the first term is the effect of the primordial non-Gaussianity of the "local type," whose Bardeen's curvature perturbation, $\Phi$, is written as $\Phi(\boldsymbol{x})=\phi(\boldsymbol{x})+f_{\mathrm{NL}} \phi^{2}(\boldsymbol{x})$, where $\phi$ is a Gaussian field (Salopek \& Bond 1990; Gangui et al. 1994; Verde et al. 2000; Komatsu \& Spergel 2001). The latest limit on this parameter is $f_{\mathrm{NL}}=38 \pm 21$ (68\% CL; Smith et al. 2009).

However, Sefusatti \& Komatsu's equation, Equation (1), may require modifications, in light of recent analytical (Dalal et al. 2008; Matarrese \& Verde 2008; Slosar et al. 2008; Afshordi \& Tolley 2008; Taruya et al. 2008; McDonald 2008) and numerical (Dalal et al. 2008; Desjacques et al. 2008; Pillepich et al. 2008; Grossi et al. 2009) studies of the effects of primordial non-Gaussianity on the galaxy power spectrum. These studies have discovered an unexpected signature of primordial nonGaussianity in the form of a scale-dependent galaxy bias, i.e., $P_{g}(k, z)=b_{1}^{2}(z) P_{m}(k, z) \rightarrow\left[b_{1}(z)+\Delta b(k, z)\right]^{2} P_{m}(k, z)$, where

$$
\Delta b(k, z)=\frac{3\left(b_{1}(z)-1\right) f_{\mathrm{NL}} \Omega_{m} H_{0}^{2} \delta_{c}}{D(z) k^{2} T(k)},
$$

and $\delta_{c} \simeq 1.68$ is the threshold linear density contrast for a spherical collapse of an overdensity region.

Then, several questions arise: can we still use Equation (1) for the bispectrum? Should we replace $b_{1}$ by $b_{1}+\Delta b(k)$ ? Does the first line in Equation (1) somehow give the same correction as $\Delta b(k)$ ? How about $b_{2}$ ? We are going to address these questions in this paper.

\section{BISPECTRUM OF DARK MATTER HALOS}

In this section, we derive the galaxy bispectrum for nonGaussian initial conditions by using two different methods. In Section 2.1, we shall use the "functional integration method" for computing $n$-point correlation functions of peaks of the cosmological density fluctuations (Politzer \& Wise 1984; Grinstein $\&$ Wise 1986). In Section 2.2, we shall present an alternative derivation of the same result by using a local bias assumption.

\subsection{Mararrese-Lucchin-Bonometto (MLB) Method}

We shall use the Matarrese-Lucchin-Bonometto (MLB) formula (Matarrese et al. 1986) which allows one to calculate the $n$-point correlation functions of peaks for non-Gaussian initial conditions. This approach is especially well suited for our purposes, as Matarrese \& Verde (2008) have applied the MLB formula to compute the scale-dependent bias of the galaxy power spectrum. We shall apply the MLB formula to compute the galaxy bispectrum for general non-Gaussian initial condition.

We study the three-point correlation function of the spatial distribution of dark matter halos. Let us consider the probability of finding three halos within three arbitrary volume elements: $d V_{1}, d V_{2}$, and $d V_{3}$, which are at $\boldsymbol{x}_{1}, \boldsymbol{x}_{2}$, and $\boldsymbol{x}_{3}$, respectively, as (Peebles 1980)

$$
\begin{aligned}
P\left(\boldsymbol{x}_{1}, \boldsymbol{x}_{2}, \boldsymbol{x}_{3}\right)= & \bar{n}^{3}\left[1+\xi_{h}\left(x_{12}\right)+\xi_{h}\left(x_{23}\right)+\xi_{h}\left(x_{31}\right)\right. \\
& \left.+\zeta_{h}\left(\boldsymbol{x}_{1}, \boldsymbol{x}_{2}, \boldsymbol{x}_{3}\right)\right] d V_{1} d V_{2} d V_{3},
\end{aligned}
$$

where $x_{i j} \equiv\left|\boldsymbol{x}_{i}-\boldsymbol{x}_{j}\right|$, and $\xi_{h}$ and $\zeta_{h}$ are the two- and three-point correlation functions of halos, respectively.

The next step is to relate the correlation functions of halos, $\xi_{h}$ and $\zeta_{h}$, to those of the underlying matter distribution function. The locations of halos coincide with those of the peaks of the matter density fluctuations; thus, one can compute $\xi_{h}$ and $\zeta_{h}$ by computing the correlation functions of peaks above a certain threshold, above which the peaks collapse into halos.

We shall assume that halos would be formed in the region where the smoothed linear density contrast exceeds $\delta_{c}$. For a spherical collapse in an Einstein-de Sitter universe $\delta_{c}=$ $3(12 \pi)^{2 / 3} / 20 \simeq 1.68$, and one can find other values in the ellipsoidal collapse in arbitrary cosmological models (see, e.g., Cooray \& Sheth 2002, for a review). The mass of halos is determined by the smoothing radius, $R$, i.e., $M=(4 \pi / 3) \rho_{m} R^{3}$, where $\rho_{m}$ is the average mass density of the universe. The smoothed density contrast, $\delta_{R}$, is related to the underlying mass fluctuations, $\delta_{m}$, as $\delta_{R}(\boldsymbol{x})=\int d^{3} \boldsymbol{x}^{\prime} W_{R}\left(\left|\boldsymbol{x}-\boldsymbol{x}^{\prime}\right|\right) \delta_{m}\left(\boldsymbol{x}^{\prime}\right)$, where $W_{R}(x)$ is a smoothing function. We shall use a top-hat filter with radius $R$ for $W_{R}(x)$.

Using the MLB formula, we find

$$
\begin{aligned}
& 1+\xi_{h}\left(x_{12}\right)+\xi_{h}\left(x_{23}\right)+\xi_{h}\left(x_{31}\right)+\zeta_{h}\left(\boldsymbol{x}_{1}, \boldsymbol{x}_{2}, \boldsymbol{x}_{3}\right) \\
& =\exp \left[\frac{1}{2} \frac{v^{2}}{\sigma_{R}^{2}} \sum_{i \neq j} \xi_{R}^{(2)}\left(x_{i j}\right)+\sum_{n=3}^{\infty}\left\{\sum_{m_{1}=0}^{n} \sum_{m_{2}=0}^{n-m_{1}} \frac{v^{n} \sigma_{R}^{-n}}{m_{1} ! m_{2} ! m_{3} !}\right.\right. \\
& \times \xi_{R}^{(n)}\left(\begin{array}{ccc}
\boldsymbol{x}_{1}, \ldots, \boldsymbol{x}_{1}, & \boldsymbol{x}_{2}, \ldots, \boldsymbol{x}_{2}, & \boldsymbol{x}_{3}, \ldots, \boldsymbol{x}_{3} \\
m_{1} \text { times } & m_{2} \text { times } & m_{3} \text { times }
\end{array}\right) \\
& \left.\left.-3 \frac{v^{n} \sigma_{R}^{-n}}{n !} \xi_{R}^{(n)}\left(\begin{array}{c}
\boldsymbol{x}, \ldots, \boldsymbol{x} \\
n \text { times }
\end{array}\right)\right\}\right] \text {, }
\end{aligned}
$$

where $m_{3} \equiv n-m_{1}-m_{2}, v \equiv \delta_{c} / \sigma_{R}, \sigma_{R}^{2}$ is the variance of matter density fluctuations smoothed by a top-hat filter with radius $R$, and $\xi_{R}^{(n)}$ denotes the connected parts of the $n$-point correlation functions of the underlying matter density fields smoothed by a top-hat filter of radius $R$. Here, we have assumed that we are dealing with high density peaks, i.e., $v \gg 1$, which are equivalent to highly biased galaxies, $b_{1} \gg 1$.

As $\xi_{R}^{(n)} \ll 1$ on the large scales that we are interested in, we expand the exponential in Equation (6). We keep the terms up to the four-point function, as this term provides the dominant contribution to the three-point function. We find

$$
\begin{aligned}
\zeta_{h}\left(\boldsymbol{x}_{1}, \boldsymbol{x}_{2}, \boldsymbol{x}_{3}\right)= & \frac{v^{3}}{\sigma_{R}^{3}} \xi_{R}^{(3)}\left(\boldsymbol{x}_{1}, \boldsymbol{x}_{2}, \boldsymbol{x}_{3}\right) \\
& +\frac{v^{4}}{\sigma_{R}^{4}}\left[\xi_{R}^{(2)}\left(x_{12}\right) \xi_{R}^{(2)}\left(x_{23}\right)+(2 \text { cyclic })\right] \\
& +\frac{v^{4}}{2 \sigma_{R}^{4}}\left[\xi_{R}^{(4)}\left(\boldsymbol{x}_{1}, \boldsymbol{x}_{1}, \boldsymbol{x}_{2}, \boldsymbol{x}_{3}\right)+(2 \text { cyclic })\right] .
\end{aligned}
$$

The bispectrum of halos in Lagrangian space, $B_{h}^{L}\left(\boldsymbol{k}_{1}, \boldsymbol{k}_{2}, \boldsymbol{k}_{3}\right)$, is the Fourier transform of $\zeta_{h}\left(x_{1}, x_{2}, x_{3}\right)$,

$$
\begin{aligned}
& B_{h}^{L}\left(\boldsymbol{k}_{1}, \boldsymbol{k}_{2}, \boldsymbol{k}_{3}\right)=\frac{v^{3}}{\sigma_{R}^{3}}\left[B_{R}\left(\boldsymbol{k}_{1}, \boldsymbol{k}_{2}, \boldsymbol{k}_{3}\right)+\frac{v}{\sigma_{R}}\right. \\
& \quad \times\left\{P_{R}\left(k_{1}\right) P_{R}\left(k_{2}\right)+(2 \text { cyclic })\right\}+\frac{v}{2 \sigma_{R}} \\
& \left.\quad \times \int \frac{d^{3} q}{(2 \pi)^{3}} T_{R}\left(\boldsymbol{q}, \boldsymbol{k}_{1}-\boldsymbol{q}, \boldsymbol{k}_{2}, \boldsymbol{k}_{3}\right)+(2 \text { cyclic })\right],
\end{aligned}
$$


where $T_{R}$ is the trispectrum, Fourier transform of $\xi_{R}^{(4)}$. Here, we call $B_{h}^{L}$ the Lagrangian space bispectrum, as it relates the halo overdensity to the initial matter overdensity with its amplitude extrapolated to the present epoch. If we assume that the halos move in the same way as matter, the observed bispectrum in Eulerian space, $B_{h}$, would be the same expression with Equation (8), except for the coefficients

$$
\begin{aligned}
& B_{h}\left(\boldsymbol{k}_{1}, \boldsymbol{k}_{2}, \boldsymbol{k}_{3}\right)=b_{1}^{3}\left[B_{R}\left(\boldsymbol{k}_{1}, \boldsymbol{k}_{2}, \boldsymbol{k}_{3}\right)\right. \\
& +\frac{b_{2}}{b_{1}}\left\{P_{R}\left(k_{1}\right) P_{R}\left(k_{2}\right)+(2 \text { cyclic })\right\} \\
& \left.+\frac{\delta_{c}}{2 \sigma_{R}^{2}} \int \frac{d^{3} q}{(2 \pi)^{3}} T_{R}\left(\boldsymbol{q}, \boldsymbol{k}_{1}-\boldsymbol{q}, \boldsymbol{k}_{2}, \boldsymbol{k}_{3}\right)+(2 \text { cyclic })\right] .
\end{aligned}
$$

Here, $b_{1}$ is the so-called linear Eulerian bias parameter, $b_{1}=1+v / \sigma_{R}$, and $b_{2}=\left(v / \sigma_{R}\right)^{2}$ is the non-linear bias parameter. $^{2}$

\subsection{Alternative Derivation}

In this section, we present an alternative derivation of the galaxy bispectrum, Equation (9). On large enough scales, we may approximate the relation between the galaxy distribution and the underlying density fluctuation as a local function. We then Taylor-expand this local function in a power series of $\delta_{m}$ (see Equation (3)).

When computing the correlation functions of halos of a given mass $M$, we may smooth the matter density field with the same filter over the corresponding length scale $R, W_{R}\left(\left|\boldsymbol{x}-\boldsymbol{x}^{\prime}\right|\right)$, which was defined in the previous section. We then Taylor-expand $\delta_{g}$ in a power series of the smoothed density field, $\delta_{R}(\boldsymbol{x})$, as

$$
\delta_{g}(\boldsymbol{x})=b_{1} \delta_{R}(\boldsymbol{x})+\frac{b_{2}}{2}\left[\delta_{R}^{2}(\boldsymbol{x})-\sigma_{R}^{2}\right]+\cdots
$$

In Fourier space, one finds

$$
\begin{aligned}
\delta_{g}(\boldsymbol{k})= & b_{1} \delta_{R}(\boldsymbol{k})+\frac{b_{2}}{2}\left[\int \frac{d^{3} q}{(2 \pi)^{3}} \delta_{R}(\boldsymbol{k}-\boldsymbol{q}) \delta_{R}(\boldsymbol{q})-\sigma_{R}^{2} \delta^{D}(\boldsymbol{k})\right] \\
& +\cdots,
\end{aligned}
$$

where $\delta^{D}$ is the Dirac delta function. We calculate the bispectrum of galaxies directly from Equation (11):

$$
\begin{aligned}
& \left\langle\delta_{g}\left(\boldsymbol{k}_{1}\right) \delta_{g}\left(\boldsymbol{k}_{2}\right) \delta_{g}\left(\boldsymbol{k}_{3}\right)\right\rangle=b_{1}^{3}\left\langle\delta_{R}\left(\boldsymbol{k}_{1}\right) \delta_{R}\left(\boldsymbol{k}_{2}\right) \delta_{R}\left(\boldsymbol{k}_{3}\right)\right\rangle \\
& +\frac{b_{1}^{2} b_{2}}{2}\left[\int \frac{d^{3} q}{(2 \pi)^{3}}\left\langle\delta_{R}\left(\boldsymbol{k}_{1}-\boldsymbol{q}\right) \delta_{R}(\boldsymbol{q}) \delta_{R}\left(\boldsymbol{k}_{2}\right) \delta_{R}\left(\boldsymbol{k}_{3}\right)\right\rangle\right. \\
& \left.\quad-\sigma_{R}^{2} \delta^{D}\left(\boldsymbol{k}_{1}\right)\left\langle\delta_{R}\left(\boldsymbol{k}_{2}\right) \delta_{R}\left(\boldsymbol{k}_{3}\right)\right\rangle+(2 \text { cyclic })\right] .
\end{aligned}
$$

The first term of Equation (12) is the matter bispectrum,

$$
\left\langle\delta_{R}\left(\boldsymbol{k}_{1}\right) \delta_{R}\left(\boldsymbol{k}_{2}\right) \delta_{R}\left(\boldsymbol{k}_{3}\right)\right\rangle=(2 \pi)^{3} B_{R}\left(k_{1}, k_{2}, k_{3}\right) \delta^{D}\left(\boldsymbol{k}_{123}\right),
$$

where $\boldsymbol{k}_{123} \equiv \boldsymbol{k}_{1}+\boldsymbol{k}_{2}+\boldsymbol{k}_{3}$. We further calculate the ensemble average of the four-point function in the second term of Equation (12). For non-Gaussian density fields, four-point

\footnotetext{
2 Note that these expressions, $b_{1}=1+\nu / \sigma_{R}$ and $b_{2}=\left(\nu / \sigma_{R}\right)^{2}$ agree with the linear halo bias parameter derived by Mo \& White (1996) and Mo et al. (1997), $b_{1}=1+b_{1}^{L}$ and $b_{2}=\left(v^{2}-3\right) / \sigma_{R}^{2}+8 b_{1}^{L} / 21$ for high-density peaks, $v \gg 1$. Here, $b_{1}^{L}=(v-1 / v) / \sigma_{R}$ is the Lagrangian bias parameter.
}

function is given by a sum of connected (trispectrum) and unconnected (products of the power spectra) parts as

$$
\begin{aligned}
& \left\langle\delta_{R}\left(\boldsymbol{k}_{1}-\boldsymbol{q}\right) \delta_{R}(\boldsymbol{q}) \delta_{R}\left(\boldsymbol{k}_{2}\right) \delta_{R}\left(\boldsymbol{k}_{3}\right)\right\rangle=(2 \pi)^{6} P_{R}(q) P_{R}\left(k_{2}\right) \\
& \quad \times \delta^{D}\left(\boldsymbol{k}_{1}\right) \delta^{D}\left(\boldsymbol{k}_{2}+\boldsymbol{k}_{3}\right)+(2 \pi)^{6} P_{R}\left(k_{2}\right) P_{R}\left(k_{3}\right) \delta^{D}\left(\boldsymbol{k}_{2}+\boldsymbol{q}\right) \\
& \quad \times \delta^{D}\left(\boldsymbol{k}_{3}+\boldsymbol{k}_{1}-\boldsymbol{q}\right)+(2 \pi)^{6} P_{R}\left(k_{2}\right) P_{R}\left(k_{3}\right) \delta^{D}\left(\boldsymbol{k}_{3}+\boldsymbol{q}\right) \\
& \quad \times \delta^{D}\left(\boldsymbol{k}_{2}+\boldsymbol{k}_{1}-\boldsymbol{q}\right)+(2 \pi)^{3} T_{R}\left(\boldsymbol{k}_{1}-\boldsymbol{q}, \boldsymbol{q}, \boldsymbol{k}_{2}, \boldsymbol{k}_{3}\right) \delta^{D}\left(\boldsymbol{k}_{123}\right),
\end{aligned}
$$

where $T_{R}$ is the matter trispectrum. Note that the first term in the above equation cancels the last term in Equation (12). Combining the above equations, Equation (12) becomes

$$
\begin{aligned}
& B_{h}\left(\boldsymbol{k}_{1}, \boldsymbol{k}_{2}, \boldsymbol{k}_{3}\right)=b_{1}^{3}\left[B_{R}\left(\boldsymbol{k}_{1}, \boldsymbol{k}_{2}, \boldsymbol{k}_{3}\right)+\frac{b_{2}}{b_{1}}\left\{P_{R}\left(k_{1}\right) P_{R}\left(k_{2}\right)\right.\right. \\
& +(2 \text { cyclic })\}+\frac{1}{2} \frac{b_{2}}{b_{1}} \int \frac{d^{3} q}{(2 \pi)^{3}} T_{R}\left(\boldsymbol{q}, \boldsymbol{k}_{1}-\boldsymbol{q}, \boldsymbol{k}_{2}, \boldsymbol{k}_{3}\right) \\
& +(2 \text { cyclic })] .
\end{aligned}
$$

Therefore, we find that the MLB method and the locality bias assumption give formally the same results.

Although the Equations (9) and (13) are the same, there is a subtle difference between them: the coefficient in the last term of Equation (13) does not include $\sigma_{R}^{2}$ explicitly. By evaluating the last cyclic term in Equation (13) for the local type of non-Gaussianity, we find that the integration of the smoothed trispectrum depends on the smoothing scale, $R$, up to a constant factor of $1 / \sigma_{R}^{2}$ on large scales, say, $k<0.1 h \mathrm{Mpc}^{-1}$. For example, the bottom right panel of Figure 11 shows that $B_{f_{\mathrm{NL}}^{2}}^{n G}$ and $B_{g_{\mathrm{NL}}}^{n G}$, which are defined in Equations (29) and (30), respectively, do not depend on the smoothing scale $R$, as they include $1 / \sigma_{R}^{2}$ in their definitions.

Therefore, it is physically more sensible to include $\sigma_{R}^{2}$ explicitly in the equation such that the dependence on smoothing scales on large scales can be absorbed by the bias parameters. This motivates our writing the final form of the halo bispectrum, derived from the local bias assumption, as

$$
\begin{aligned}
& B_{h}\left(\boldsymbol{k}_{1}, \boldsymbol{k}_{2}, \boldsymbol{k}_{3}\right)=b_{1}^{3}\left[B_{R}\left(\boldsymbol{k}_{1}, \boldsymbol{k}_{2}, \boldsymbol{k}_{3}\right)+\frac{b_{2}}{b_{1}}\left\{P_{R}\left(k_{1}\right) P_{R}\left(k_{2}\right)\right.\right. \\
& +(2 \text { cyclic })\}+\frac{\tilde{b}_{2}}{b_{1}} \frac{1}{2 \sigma_{R}^{2}} \int \frac{d^{3} q}{(2 \pi)^{3}} T_{R}\left(\boldsymbol{q}, \boldsymbol{k}_{1}-\boldsymbol{q}, \boldsymbol{k}_{2}, \boldsymbol{k}_{3}\right) \\
& \quad+(2 \text { cyclic })]
\end{aligned}
$$

with three bias parameters: $b_{1}, b_{2}$ and $\tilde{b}_{2} \equiv b_{2} \sigma_{R}^{2}$. Note that $\tilde{b}_{2} / b_{1} \rightarrow \delta_{c}$ for the MLB formula. Although $\delta_{c}$ is known to be 1.68 for the spherically collapsed halo in the flat matter dominated universe, its precise value, in this context, needs to be tested against $N$-body simulations.

Equations (9) and (14) are the first main results of this paper, which are general and can be applied to any models of nonGaussianities, once we know the bispectrum and trispectrum of the underlying matter density field. Note that Equation (14) was also obtained independently by Sefusatti (2009).

In principle both $b_{1}$ and $b_{2}$ are calculable from the theory of collapse of halos (see, e.g., Cooray \& Sheth 2002, for a review); however, in practice it is more convenient and safe to treat them as free parameters that one should marginalize over 
when extracting the cosmological information, such as $f_{\mathrm{NL}}$. See Jeong \& Komatsu (2009) for the same argument applied to the galaxy power spectrum.

\section{EFFECTS OF LOCAL-TYPE PRIMORDIAL NON-GAUSSIANITY ON THE HALO BISPECTRUM}

In this section, we shall evaluate Equation (14) for the localtype primordial non-Gaussianity with a high-order term added

$$
\Phi(\boldsymbol{x})=\phi(\boldsymbol{x})+f_{\mathrm{NL}}\left[\phi^{2}(\boldsymbol{x})-\left\langle\phi^{2}\right\rangle\right]+g_{\mathrm{NL}} \phi^{3}(\boldsymbol{x}) .
$$

The cubic-order term does not generate the bispectrum of $\mathrm{CMB}$ anisotropy or the matter density fluctuations at the leading order; however, it does generate the trispectrum, and the CMB trispectrum has been calculated by Okamoto \& Hu (2002); Kogo $\&$ Komatsu (2006). On the other hand, the bispectrum of halos receives the contribution from the trispectrum (see the last term in Equation (14)), and thus it is necessary to include $g_{\mathrm{NL}}$.

To calculate various components of the bispectra shown in Equation (14), we calculate the transfer function, $T(k)$, and the power spectra with the cosmological parameters in Table 1 ("WMAP+BAO+SN") of Komatsu et al. (2009).

As for the smoothing scale, we use $R=1 h^{-1} \mathrm{Mpc}$. Although the smoothing scale explicitly appears in the equation, it makes negligible differences for the bispectrum on large scales, $k \ll 1 / R$.

Note that we shall adopt the non-standard convention in which $\Phi(x)$ is Bardeen's curvature perturbation extrapolated to the present epoch, $z=0$, using the linear growth factor of $\Phi, g(z) \equiv$ $(1+z) D(z)$. Therefore, our $f_{\mathrm{NL}}$ and $g_{\mathrm{NL}}$ in this paper are different from those in the literature on the CMB non-Gaussianity by a factor of $g(1090) / g(0)$, i.e., $f_{\mathrm{NL}}=[g(1090) / g(0)] f_{\mathrm{NL}}^{\mathrm{CMB}}$ and $g_{\mathrm{NL}}=\left[g^{2}(1090) / g^{2}(0)\right] g_{\mathrm{NL}}^{\mathrm{CMB}}$. $^{3}$

The bispectrum and trispectrum of $\Phi$ are given by

$$
B_{\Phi}\left(\boldsymbol{k}_{1}, \boldsymbol{k}_{2}, \boldsymbol{k}_{3}\right)=2 f_{\mathrm{NL}}\left[P_{\phi}\left(k_{1}\right) P_{\phi}\left(k_{2}\right)+(2 \text { cyclic })\right],
$$

and

$$
\begin{aligned}
& T_{\Phi}\left(\boldsymbol{k}_{1}, \boldsymbol{k}_{2}, \boldsymbol{k}_{3}, \boldsymbol{k}_{4}\right)=6 g_{\mathrm{NL}}\left[P_{\phi}\left(k_{1}\right) P_{\phi}\left(k_{2}\right) P_{\phi}\left(k_{3}\right)+(3 \text { cyclic })\right] \\
& \quad+2 f_{\mathrm{NL}}^{2}\left[P_{\phi}\left(k_{1}\right) P_{\phi}\left(k_{2}\right)\left\{P_{\phi}\left(k_{13}\right)+P_{\phi}\left(k_{14}\right)\right\}+(11 \text { cyclic })\right],
\end{aligned}
$$

with $k_{i j}=\left|\boldsymbol{k}_{i}+\boldsymbol{k}_{j}\right|$, respectively.

While Equation (17) is the consequence of Equation (15), general multi-field inflation models do not necessarily relate the coefficients of the trispectrum to that of the bispectrum. Therefore, one may generalize Equation (17) by replacing $f_{\mathrm{NL}}^{2}$ with a new parameter, $\tau_{\mathrm{NL}}$, which may or may not be related to $f_{\mathrm{NL}}$,

$$
\begin{array}{r}
T_{\Phi}\left(\boldsymbol{k}_{1}, \boldsymbol{k}_{2}, \boldsymbol{k}_{3}, \boldsymbol{k}_{4}\right)=6 g_{\mathrm{NL}}\left[P_{\phi}\left(k_{1}\right) P_{\phi}\left(k_{2}\right) P_{\phi}\left(k_{3}\right)+(3 \text { cyclic })\right] \\
\quad+\frac{25}{18} \tau_{\mathrm{NL}}\left[P_{\phi}\left(k_{1}\right) P_{\phi}\left(k_{2}\right)\left\{P_{\phi}\left(k_{13}\right)+P_{\phi}\left(k_{14}\right)\right\}+(11 \text { cyclic })\right] .
\end{array}
$$

Note that the coefficient of $\tau_{\mathrm{NL}}$ reflects the definition of $\tau_{\mathrm{NL}}$ introduced by Boubekeur \& Lyth (2006). This opens up an exciting possibility that the galaxy bispectrum can test whether

\footnotetext{
3 The ratio $g(1090) / g(0)$ is 1.308 for the cosmological parameters in Table 1 (“WMAP+BAO+SN") of Komatsu et al. (2009).
}

$\tau_{\mathrm{NL}}=\left(6 f_{\mathrm{NL}} / 5\right)^{2}$ or other predictions for the relation between $\tau_{\mathrm{NL}}$ and $f_{\mathrm{NL}}$ are satisfied observationally.

We transform these spectra to those of the smoothed linear density contrasts, using the Poisson equation,

$$
\delta_{R}^{(1)}(k)=\frac{2}{3} \frac{k^{2} T(k)}{H_{0}^{2} \Omega_{m}} \tilde{W}_{R}(k) \Phi(k) \equiv \mathcal{M}_{R}(k) \Phi(k),
$$

where $\tilde{W}_{R}(k)$ is the Fourier transform of the top-hat filter with radius $R$. Note that $\tilde{W}_{R}(k) \rightarrow 1$ as $k \rightarrow 0$. In general, $\tilde{W}_{R}(k) \simeq 1$ for $k \ll 1 / R$. Then, we calculate the $n$-point function of the matter density fields from the corresponding correlator of curvature perturbations by

$$
\left\langle\delta_{R}^{(1)}\left(\boldsymbol{k}_{1}\right) \cdots \delta_{R}^{(1)}\left(\boldsymbol{k}_{n}\right)\right\rangle=\prod_{i=1}^{n} \mathcal{M}_{R}\left(k_{i}\right)\left\langle\Phi\left(\boldsymbol{k}_{1}\right) \cdots \Phi\left(\boldsymbol{k}_{n}\right)\right\rangle .
$$

\subsection{Known Terms}

\subsubsection{Formula}

The first term in Equation (14) contains the bispectrum of matter density fluctuations, $B_{R}$, which consists of two pieces: (1) the non-linear evolution of gravitational clustering $\left(B_{m}^{G}\right)$ and (2) primordial non-Gaussianity $\left(B_{f_{\mathrm{NL}}}^{n G 0}\right)^{4}$,

$$
B_{R}\left(k_{1}, k_{2}, k_{3}\right)=B_{m}^{G}\left(k_{1}, k_{2}, k_{3}\right)+f_{\mathrm{NL}} B_{f_{\mathrm{NL}}}^{n G 0}\left(k_{1}, k_{2}, k_{3}\right),
$$

where

$$
\begin{aligned}
B_{m}^{G}\left(k_{1}, k_{2}, k_{3}\right) \equiv & \tilde{W}_{R}\left(k_{1}\right) \tilde{W}_{R}\left(k_{2}\right) \tilde{W}_{R}\left(k_{3}\right) 2 F_{2}^{(s)}\left(\boldsymbol{k}_{1}, \boldsymbol{k}_{2}\right) \\
& \times P_{m}\left(k_{1}\right) P_{m}\left(k_{2}\right)+(2 \text { cyclic }),
\end{aligned}
$$

with $F_{2}^{(s)}$ given by Equation (2), and

$$
\begin{aligned}
B_{f_{\mathrm{NL}}}^{n G 0}\left(k_{1}, k_{2}, k_{3}\right) & \equiv 2 \prod_{i=1}^{3} \mathcal{M}_{R}\left(k_{i}\right)\left[P_{\phi}\left(k_{1}\right) P_{\phi}\left(k_{2}\right)+(2 \text { cyclic })\right] \\
& =2 \frac{P_{R}\left(k_{1}\right)}{\mathcal{M}_{R}\left(k_{1}\right)} \frac{P_{R}\left(k_{2}\right)}{\mathcal{M}_{R}\left(k_{2}\right)} \mathcal{M}_{R}\left(k_{3}\right)+(2 \text { cyclic })
\end{aligned}
$$

One finds that Equations (22) and (21) agree with the first and the second terms in Equation (1) on the scales much larger than the smoothing scale, i.e., $k \ll 1 / R$, for which $\tilde{W}_{R} \rightarrow 1$.

One might wonder if it is OK to include the bispectrum from non-linear evolution of density fluctuations in the MLB formula, as Equation (9) is usually used for the Lagrangian density fluctuations, i.e., "initial" fluctuations. However, it is perfectly OK to use the evolved density fluctuations in this formula, as one can always use the evolved density fluctuations as the initial data. For example, we can think of starting our calculation at $z=10$, and ask the MLB formula to take the initial condition at $z=10$, including non-linear correction. Since we know how to compute the bispectrum, trispectrum, etc., of the underlying

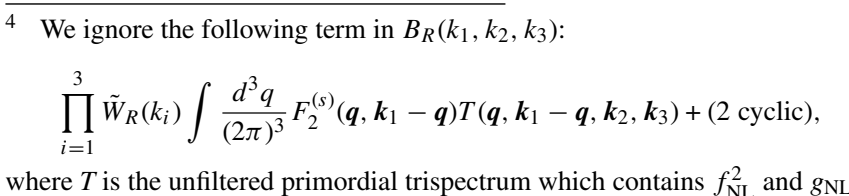
where $T$ is the unfiltered primordial trispectrum which con
This term is negligibly small (Scoccimarro et al. 2004). 
(a) squeezed triangle $\left(k_{1} \simeq k_{2}>>k_{3}\right)$

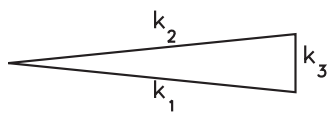

(d) isosceles triangle $\left(k_{1}>k_{2}=k_{3}\right)$

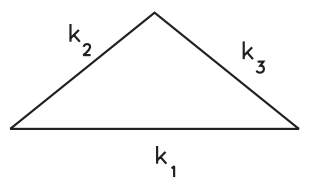

(b) elongated triangle $\left(k_{1}=k_{2}+k_{3}\right)$

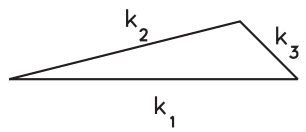

(e) equilateral triangle

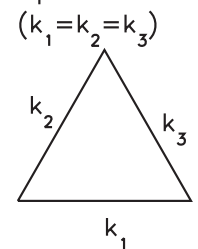

(c) folded triangle $\left(k_{1}=2 k_{2}=2 k_{3}\right)$
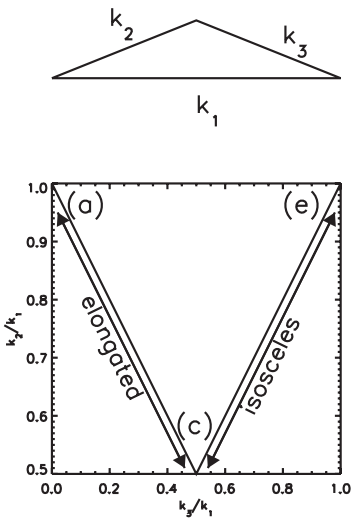

Figure 1. Visual representations of triangles forming the bispectrum, $B\left(k_{1}, k_{2}, k_{3}\right)$, with various combinations of wavenumbers satisfying $k_{3} \leqslant k_{2} \leqslant k_{1}$.
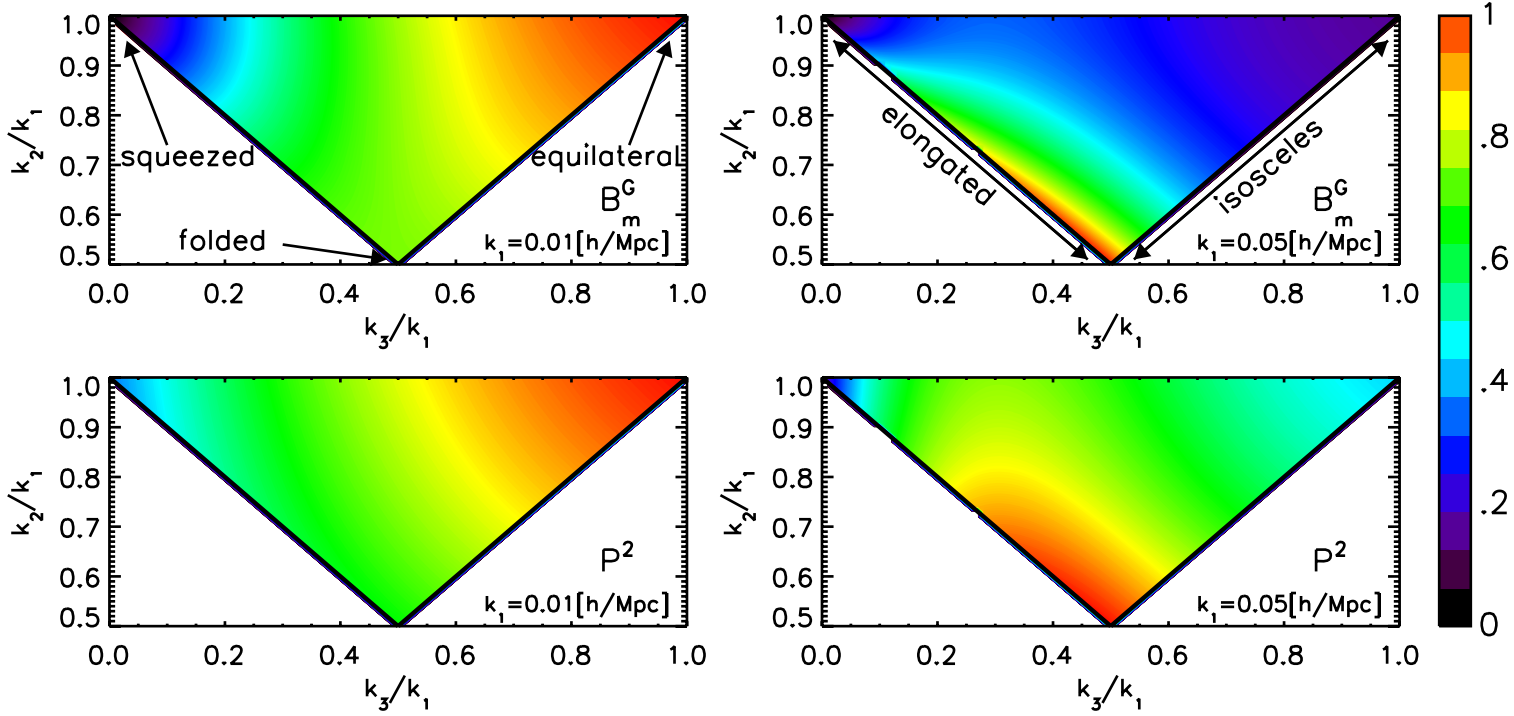

Figure 2. Shape of the bispectrum, $B\left(k_{1}, k_{2}, k_{3}\right)$. Each panel shows the amplitude of the bispectrum as a function of $k_{2} / k_{1}$ and $k_{3} / k_{1}$ for a given $k_{1}$, with a condition that $k_{3} \leqslant k_{2} \leqslant k_{1}$ is satisfied. The amplitude is normalized such that it is unity at the point where the bispectrum takes on the maximum value. For the visual representations of the triangle names such as the squeezed, elongated, folded, isosceles, and equilateral, see Figure 1. Top Left: the bispectrum from the non-linear gravitational evolution, $B_{m}^{G}$ (Equation (21)), for $k_{1}=0.01 h \mathrm{Mpc}^{-1}$. Top Right: $B_{m}^{G}$ for $k_{1}=0.05 h \mathrm{Mpc}^{-1}$. Bottom Left: the bispectrum from the non-linear galaxy biasing, $P_{R}\left(k_{1}\right) P_{R}\left(k_{2}\right)+(2 \mathrm{cyclic})$ (the second term in Equation (14)), for $k_{1}=0.01 h \mathrm{Mpc}^{-1}$. (Bottom Right) $P_{R}\left(k_{1}\right) P_{R}\left(k_{2}\right)+(2 \mathrm{cyclic})$ for $k_{1}=0.05 h \mathrm{Mpc}^{-1}$.

(A color version of this figure is available in the online journal.)

mass distribution at $z=10$ (including non-linear effects), we can use this information in the MLB formula. In other words, the "initial" distribution does not need to be primordial. We can provide the evolved density field as the initial data, and compute the peak statistics. The MLB formula does not care whether the source of non-Gaussianity is truly primordial or not: the only conditions that we must respect for Equation (9) to be valid are (1) high peaks $(v \gg 1)$ and (2) $n$-point correlation functions are much less than unity, $\xi_{R}^{(n)} \ll 1$, so that the exponential in Equation (6) can be Taylor expanded. In this case, one would lose an ability to calculate the bias parameters, $b_{1}$ and $b_{2}$, using, e.g., a halo model; however, this is not a disadvantage, as the halo model calculations of the galaxy bias parameter, $b_{1}$ and $b_{2}$, are at best qualitative even for Gaussian initial conditions (see, e.g., Jeong \& Komatsu 2009). In our approach, the coefficients of individual terms in Equations (9) and (14), including $\delta_{c}$, are free parameters, and need to be determined from observations themselves.

\subsubsection{Shape Dependence: Non-linear Gravitational Evolution and Non-linear Galaxy Bias}

How about the shape dependence? First, let us review the structure of $B_{m}^{G}\left(k_{1}, k_{2}, k_{3}\right)$ (Equation (21)), which has been studied extensively in the literature (see Bernardeau et al. 2002, for a review).

Here, let us offer a useful way of visualizing the shape dependence of the bispectrum. We can study the structure of the bispectrum by plotting the magnitude of $B_{m}^{G}$ as a function of $k_{2} / k_{1}$ and $k_{3} / k_{1}$ for a given $k_{1}$, with a condition that $k_{1} \geqslant k_{2} \geqslant k_{3}$ is satisfied. In order to classify various shapes of the triangles, let us use the following names: squeezed $\left(k_{1} \simeq\right.$ $\left.k_{2} \gg k_{3}\right)$, elongated $\left(k_{1}=k_{2}+k_{3}\right)$, folded $\left(k_{1}=2 k_{2}=2 k_{3}\right)$, isosceles $\left(k_{2}=k_{3}\right)$, and equilateral $\left(k_{1}=k_{2}=k_{3}\right)$. See panels (a)-(e) of Figure 1 for the visual representations of these triangles.

The top-left panel of Figure 2 shows $B_{m}^{G}$ for $k_{1}=$ $0.01 h \mathrm{Mpc}^{-1}$. In this regime, $P_{R}\left(k_{1}\right)$ is an increasing function 

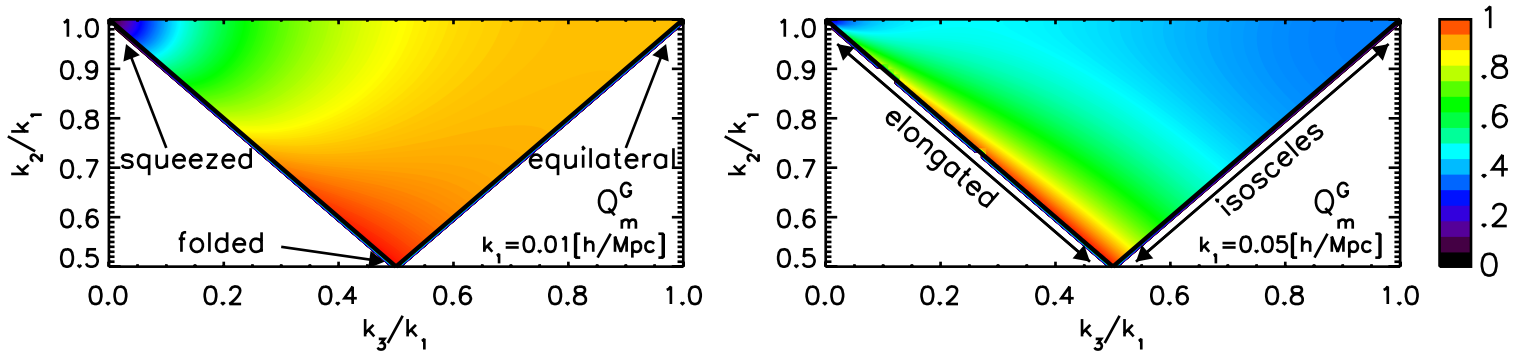

Figure 3. Same as the top panels of Figure 2, but for $B_{m}^{G} /\left[P_{R}\left(k_{1}\right) P_{R}\left(k_{2}\right)+(2\right.$ cyclic)] (Equation (23)).

(A color version of this figure is available in the online journal.)

of $k_{1}$ (recall that $P_{R}(k)$ peaks at $\left.k \approx 0.02 h \mathrm{Mpc}^{-1}\right)$. Let us then pick the first term in Equation $(21), F_{2}^{(s)}\left(\boldsymbol{k}_{1}, \boldsymbol{k}_{2}\right) P_{R}\left(k_{1}\right) P_{R}\left(k_{2}\right)$, and ignore the cyclic terms for the moment. It follows from Equation (2) and the descriptions below it that $F_{2}^{(s)}\left(\boldsymbol{k}_{1}, \boldsymbol{k}_{2}\right)$ vanishes in the squeezed limit $\left(\boldsymbol{k}_{1}=-\boldsymbol{k}_{2}\right)$ and reaches the maximum in the opposite limit $\left(\boldsymbol{k}_{1}=\alpha \boldsymbol{k}_{2}\right)$. Therefore, we would expect this term to give large signals in the "elongated configurations," $k_{1}=k_{2}+k_{3}$; however, as $P_{R}(k)$ at $k \lesssim 0.02 h \mathrm{Mpc}^{-1}$ is an increasing function of $k$, one can also get large signals when $k_{1}$ and $k_{2}$ are equally large, $k_{1}=k_{2}$. As we have zero signal in the squeezed limit, $k_{3}=0$, it follows that we can find a large signal in the equilateral configuration, $k_{1}=k_{2}=k_{3}$. A similar argument also applies to the cyclic terms. As a result, for $k_{1}=0.01 h \mathrm{Mpc}^{-1}$, we find the largest signal in the equilateral configuration, and then the signal decreases as we approach the squeezed configuration, i.e., the signal decreases as we go from panel (e) to (a) in Figure 1.

The top-right panel of Figure 2 shows $B_{m}^{G}$ for $k_{1}=$ $0.05 h \mathrm{Mpc}^{-1}$. In this regime, $P_{R}\left(k_{1}\right)$ is a decreasing function of $k_{1}$, and thus the equilateral configurations are no longer as important as the folded ones. Instead, we have large signals in the folded configurations as well as in the elongated configurations. Note that the exact squeezed limit is still suppressed due to the form of $F_{2}^{(s)}$.

In summary, $B_{m}^{G}$ usually has the largest signal in the folded and elongated (or equilateral, depending on the wavenumber) configurations, with the squeezed configurations suppressed relative to the others. The suppression of the squeezed configurations is a generic signature of the causal mechanism such as the non-linear gravitational evolution that $F_{2}^{(s)}$ describes.

The bispectrum from the non-linear bias term, the second term in Equation (9), has the same structure as $B_{m}^{G}$, but it does not contain $F_{2}^{(s)}$. As a result the non-linear bias term does not have as much suppression as $B_{m}^{G}$ has in the squeezed configuration. In addition, as $F_{2}^{(s)}$ enhances the signal in the elongated configurations, the non-linear bias term does not have as much enhancement as $B_{m}^{G}$ has in the elongated configurations. These properties explain the bottom panels of Figure 2.

As $B_{m}^{G}$ and the non-linear bias term contain products of $P_{R}\left(k_{1}\right) P_{R}\left(k_{2}\right)$ and the cyclic terms, it is often more convenient to deal with $Q_{h}\left(k_{1}, k_{2}, k_{3}\right)$ given by Peebles (1980),

$$
Q_{h}\left(k_{1}, k_{2}, k_{3}\right) \equiv \frac{B_{h}\left(k_{1}, k_{2}, k_{3}\right)}{P_{R}\left(k_{1}\right) P_{R}\left(k_{2}\right)+(2 \text { cyclic })},
$$

to reduce the dependence on the shape of the power spectrum. This combination is constant and equal to $b_{1}^{2} b_{2}$ for the non-linear bias term (see the second term in Equation (9)).

The left and right panels of Figure 3 show $B_{m}^{G}\left(k_{1}, k_{2}, k_{3}\right) /$ $\left[P_{R}\left(k_{1}\right) P_{R}\left(k_{2}\right)+(2\right.$ cyclic $\left.)\right]$ for $k_{1}=0.01 h \mathrm{Mpc}^{-1}$ and
$0.05 h \mathrm{Mpc}^{-1}$, respectively. We find that $Q_{h}$ better reflects the shape dependence of $F_{2}^{(s)}$ irrespective of $k_{1}$ : it has the largest signal in the folded and elongated configurations in both large and small scales. The squeezed configurations are still heavily suppressed relative to the others.

\subsubsection{Shape Dependence: $f_{\mathrm{NL}}$ Term}

How about the $f_{\mathrm{NL}}$ term, $B_{f_{\mathrm{NL}}}^{n G 0}\left(k_{1}, k_{2}, k_{3}\right)$ ? This term has a completely different structure. Let us pick the first term, $\mathcal{M}_{R}\left(k_{1}\right) P_{\phi}\left(k_{1}\right) \mathcal{M}_{R}\left(k_{2}\right) P_{\phi}\left(k_{2}\right) \mathcal{M}_{R}\left(k_{3}\right)$, in Equation (22). The important point is that the power spectrum of $\phi$ is always a decreasing function of $k$, i.e., $P_{\phi}(k) \propto 1 / k^{3}$ for a scale-invariant spectrum. On the other hand, on large scales, we have $T(k) \rightarrow 1$ and $\mathcal{M}_{R}(k) \propto k^{2}$. Therefore, collecting all the cyclic terms, we find $B_{f_{\mathrm{NL}}}^{n G 0}\left(k_{1}, k_{2}, k_{3}\right) \propto k_{3}^{2} /\left(k_{1} k_{2}\right)+k_{2}^{2} /\left(k_{1} k_{3}\right)+k_{1}^{2} /\left(k_{2} k_{3}\right)=$ $\left(k_{1}^{3}+k_{2}^{3}+k_{3}^{3}\right) /\left(k_{1} k_{2} k_{3}\right)$. In other words, it has the largest signal when one of $k$ 's is very small, i.e., the squeezed configurations, which is opposite to the structures of $B_{m}^{G}$ and the nonlinear bias term. The middle panels of Figure 4 show $B_{f_{\mathrm{NL}}}^{n G 0}$ for $k_{1}=0.01 h \mathrm{Mpc}^{-1}$ and $0.05 h \mathrm{Mpc}^{-1}$, and we find the largest signals in the squeezed configurations. We also find that $Q_{h}$ from the $f_{\mathrm{NL}}$ term, $B_{f_{\mathrm{NL}}}^{n G 0}\left(k_{1}, k_{2}, k_{3}\right) /\left[P_{R}\left(k_{1}\right) P_{R}\left(k_{2}\right)+\right.$ (2cyclic)], still has the largest signal in the squeezed configurations.

These properties allow us to distinguish between the primordial non-Gaussianity and the other effects such as the non-linear gravitational evolution and non-linear bias. Sefusatti \& Komatsu (2007) have studied in detail how well one can separate these effects using $Q_{h}$.

\subsection{New Term}

\subsubsection{Formula}

Now, we shall evaluate the new term, the third term in Equation (14), which was not considered in Sefusatti \& Komatsu (2007). The trispectrum is generated by the primordial nonGaussianity, as well as by the non-linear evolution of matter density fluctuations. The non-linear evolution of matter density fluctuations on large scales is given by perturbation theory (see Bernardeau et al. 2002, for a review). Let us expand the filtered non-linear matter density field in Fourier space as

$$
\delta_{R}(\boldsymbol{k})=\tilde{W}_{R}(k)\left[\delta^{(1)}(\boldsymbol{k})+\delta^{(2)}(\boldsymbol{k})+\delta^{(3)}(\boldsymbol{k})+\cdots\right],
$$

where $\delta^{(n)}(\boldsymbol{k})$ is the $n$th order quantity of the linear density contrast, $\delta^{(1)}(\boldsymbol{k})$. Then, the connected matter density trispectrum is given by

$$
\begin{aligned}
& T_{R}\left(\boldsymbol{k}_{1}, \boldsymbol{k}_{2}, \boldsymbol{k}_{3}, \boldsymbol{k}_{4}\right) \\
& \quad=T_{R}^{1111}\left(\boldsymbol{k}_{1}, \boldsymbol{k}_{2}, \boldsymbol{k}_{3}, \boldsymbol{k}_{4}\right)+\left\{T_{R}^{1112}\left(\boldsymbol{k}_{1}, \boldsymbol{k}_{2}, \boldsymbol{k}_{3}, \boldsymbol{k}_{4}\right)+(3 \text { cyclic })\right\}
\end{aligned}
$$



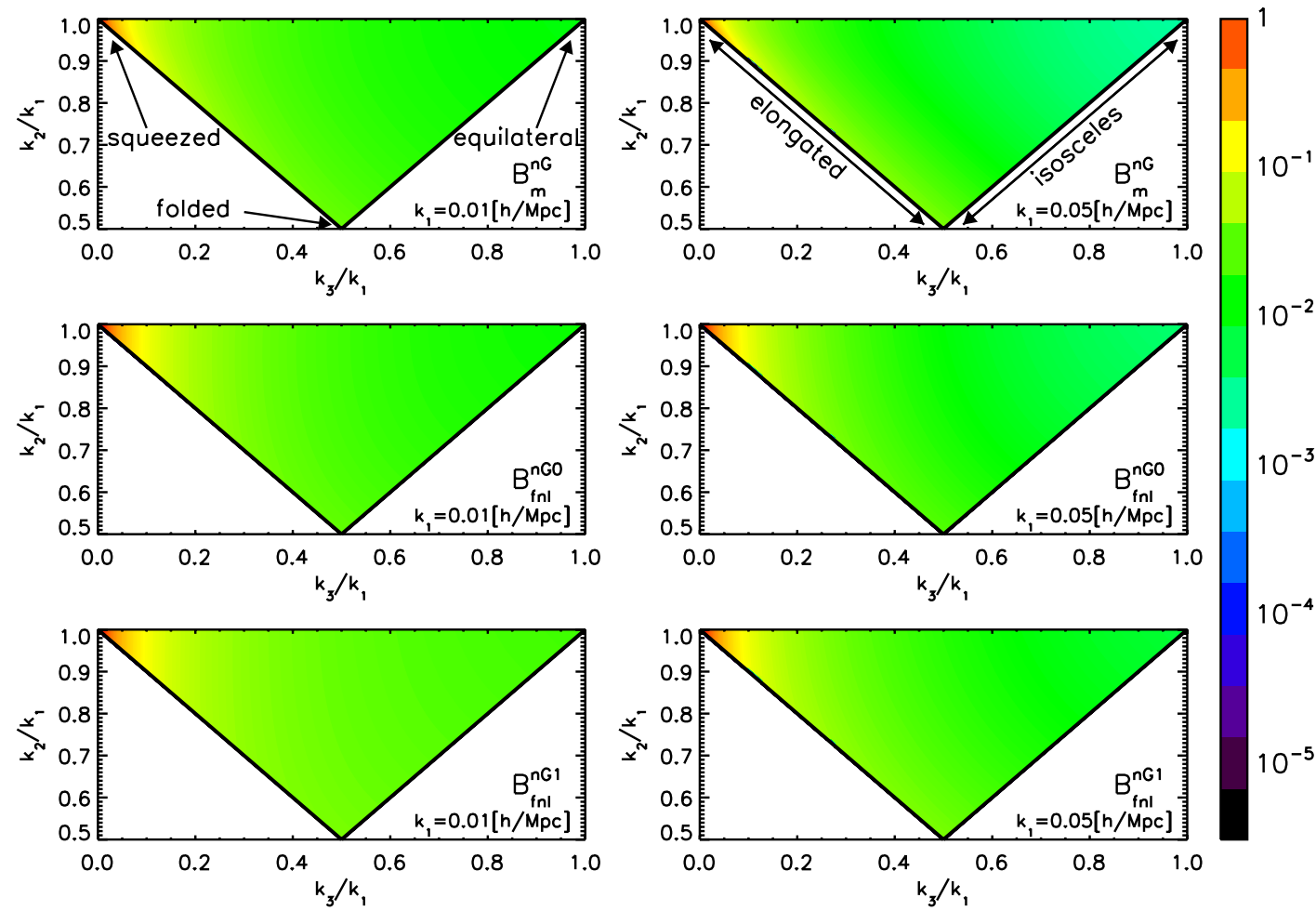

Figure 4. Same as Figure 2, but for the terms proportional to $f_{\mathrm{NL}}$. (Top) the $B_{m}^{n G}$ term (Equation (33)), (Middle) the $B_{f_{\mathrm{NL}}}^{n G 0}$ term (Equation (22)), and (Bottom) the $B_{f_{\mathrm{NL}}}^{n G 1}$ term (Equation (39)). Note that the non-Gaussian terms diverge in the exact squeezed limit, $k_{3} \rightarrow 0$; thus, we show these terms normalized to be unity at $k_{3} / k_{1}=10^{-2}$. In order to facilitate the comparison better, we draw the dotted contour for all six panels.

(A color version of this figure is available in the online journal.)

$$
\begin{aligned}
& +\left\{T_{R}^{1113}\left(\boldsymbol{k}_{1}, \boldsymbol{k}_{2}, \boldsymbol{k}_{3}, \boldsymbol{k}_{4}\right)+(3 \text { cyclic })\right\} \\
& +\left\{T_{R}^{1122}\left(\boldsymbol{k}_{1}, \boldsymbol{k}_{2}, \boldsymbol{k}_{3}, \boldsymbol{k}_{4}\right)+(5 \text { cyclic })\right\}+\mathcal{O}\left(\phi^{8}\right),
\end{aligned}
$$

with $T_{R}^{i j k l}$ given by

$$
\begin{aligned}
& (2 \pi)^{3} \delta^{D}\left(\boldsymbol{k}_{1234}\right) T_{R}^{i j k l}\left(\boldsymbol{k}_{1}, \boldsymbol{k}_{2}, \boldsymbol{k}_{3}, \boldsymbol{k}_{4}\right) \\
& \quad \equiv \prod_{n=1}^{4} \tilde{W}_{R}\left(k_{n}\right)\left\langle\delta^{(i)}\left(\boldsymbol{k}_{1}\right) \delta^{(j)}\left(\boldsymbol{k}_{2}\right) \delta^{(k)}\left(\boldsymbol{k}_{3}\right) \delta^{(l)}\left(\boldsymbol{k}_{4}\right)\right\rangle_{c} .
\end{aligned}
$$

The leading contributions of all the terms shown in Equation (25) are order of $\phi^{6}$.

The first term, $T_{R}^{1111}$, is the linearly evolved primordial trispectrum calculated from Equation (17), and thus it contains the terms proportional to $f_{\mathrm{NL}}^{2}$ and $g_{\mathrm{NL}}$. The second term, $T_{R}^{1112}$, has a coupling between the primordial non-Gaussianity (linear in $f_{\mathrm{NL}}$ ) and the non-linear gravitational evolution (linear in $F_{2}^{(s)}$ ). These two terms are important on large scales.

The other terms, $T_{R}^{1113}$ and $T_{R}^{1122}$, do not have contributions from $f_{\mathrm{NL}}$ or $g_{\mathrm{NL}}$ at the leading-order level, but solely come from the non-linear gravitational coupling; thus, they may be ignored on large scales we are considering in this paper. Sefusatti (2009) also derived and studied $T_{R}^{1112}$ as well as $T_{R}^{1113}$ and $T_{R}^{1122}$.

Therefore, we approximate the integration in the third term of Equation (14) as

$$
\begin{aligned}
& \frac{1}{2 \sigma_{R}^{2}} \int \frac{d^{3} q}{(2 \pi)^{3}}\left[T_{R}\left(\boldsymbol{q}, \boldsymbol{k}_{1}-\boldsymbol{q}, \boldsymbol{k}_{2}, \boldsymbol{k}_{3}\right)+(2 \text { cyclic })\right] \\
& \approx \\
& \quad \frac{1}{2 \sigma_{R}^{2}}\left\{\int \frac{d^{3} q}{(2 \pi)^{3}}\left[T_{R}^{(1)}\left(\boldsymbol{q}, \boldsymbol{k}_{1}-\boldsymbol{q}, \boldsymbol{k}_{2}, \boldsymbol{k}_{3}\right)+(2 \mathrm{cyclic})\right]\right. \\
& \left.\quad+\int \frac{d^{3} q}{(2 \pi)^{3}}\left[T_{R}^{(2)}\left(\boldsymbol{q}, \boldsymbol{k}_{1}-\boldsymbol{q}, \boldsymbol{k}_{2}, \boldsymbol{k}_{3}\right)+(2 \text { cyclic })\right]\right\},
\end{aligned}
$$

where "cyclic" denotes the cyclic combinations of $k_{1}, k_{2}$, and $k_{3}$, and $T_{R}^{(1)}$ and $T_{R}^{(2)}$ denote $T_{R}^{(1)}\left(\boldsymbol{k}_{1}, \boldsymbol{k}_{2}, \boldsymbol{k}_{3}, \boldsymbol{k}_{4}\right)=T_{R}^{1111}\left(\boldsymbol{k}_{1}, \boldsymbol{k}_{2}\right.$, $\left.\boldsymbol{k}_{3}, \boldsymbol{k}_{4}\right)$, and $T_{R}^{(2)}\left(\boldsymbol{k}_{1}, \boldsymbol{k}_{2}, \boldsymbol{k}_{3}, \boldsymbol{k}_{4}\right)=T_{R}^{1112}\left(\boldsymbol{k}_{1}, \boldsymbol{k}_{2}, \boldsymbol{k}_{3}, \boldsymbol{k}_{4}\right)+$ (3 cyclic), respectively.

The first term in Equation (27) is the integration of the linearly evolved primordial curvature trispectrum, which contains two pieces: one proportional to $f_{\mathrm{NL}}^{2}$ and another to $g_{\mathrm{NL}}$ (see Equation (17)). Therefore, we symbolically write the first line in Equation (27) as

$$
\begin{aligned}
& \frac{1}{2 \sigma_{R}^{2}} \int \frac{d^{3} q}{(2 \pi)^{3}}\left[T_{R}^{(1)}\left(\boldsymbol{q}, \boldsymbol{k}_{1}-\boldsymbol{q}, \boldsymbol{k}_{2}, \boldsymbol{k}_{3}\right)+(2 \text { cyclic })\right] \\
& \quad=g_{\mathrm{NL}} B_{g_{\mathrm{NL}}}^{n G}\left(k_{1}, k_{2}, k_{3}\right)+f_{\mathrm{NL}}^{2} B_{f_{\mathrm{NL}}^{2}}^{n G}\left(k_{1}, k_{2}, k_{3}\right),
\end{aligned}
$$

where

$$
\begin{aligned}
& B_{f_{\mathrm{NL}}^{2}}^{n G}\left(k_{1}, k_{2}, k_{3}\right) \equiv \frac{1}{2 \sigma_{R}^{2}}\left[4 \mathcal{M}_{R}\left(k_{2}\right) \mathcal{M}_{R}\left(k_{3}\right)\right. \\
& \quad \times \int \frac{d^{3} q}{(2 \pi)^{3}} \mathcal{M}_{R}(q) \mathcal{M}_{R}\left(\left|\boldsymbol{k}_{1}-\boldsymbol{q}\right|\right) P_{\phi}(q) \\
& \quad \times\left[P_{\phi}\left(\left|\boldsymbol{k}_{1}-\boldsymbol{q}\right|\right) P_{\phi}\left(\left|\boldsymbol{k}_{2}+\boldsymbol{q}\right|\right)+P_{\phi}\left(\left|\boldsymbol{k}_{1}-\boldsymbol{q}\right|\right) P_{\phi}\left(\left|\boldsymbol{k}_{3}+\boldsymbol{q}\right|\right)\right] \\
& \quad+(2 \mathrm{cyclic})+8 \mathcal{M}_{R}\left(k_{2}\right) \mathcal{M}_{R}\left(k_{3}\right) P_{\phi}\left(k_{2}\right) \\
& \quad \times \int \frac{d^{3} q}{(2 \pi)^{3}} \mathcal{M}_{R}(q) \mathcal{M}_{R}\left(\left|\boldsymbol{k}_{1}-\boldsymbol{q}\right|\right) P_{\phi}(q) P_{\phi}\left(\left|\boldsymbol{k}_{3}+\boldsymbol{q}\right|\right) \\
& \quad+(2 \text { cyclic })+8 \mathcal{M}_{R}\left(k_{2}\right) \mathcal{M}_{R}\left(k_{3}\right) P_{\phi}\left(k_{3}\right) \\
& \quad \times \int \frac{d^{3} q}{(2 \pi)^{3}} \mathcal{M}_{R}(q) \mathcal{M}_{R}\left(\left|\boldsymbol{k}_{1}-\boldsymbol{q}\right|\right) P_{\phi}(q) P_{\phi}\left(\left|\boldsymbol{k}_{2}+\boldsymbol{q}\right|\right) \\
& \quad+(2 \text { cyclic })+8 \mathcal{M}_{R}\left(k_{2}\right) \mathcal{M}_{R}\left(k_{3}\right) P_{\phi}\left(k_{1}\right)\left[P_{\phi}\left(k_{2}\right)+P_{\phi}\left(k_{3}\right)\right]
\end{aligned}
$$




$$
\begin{aligned}
& \quad \times \int \frac{d^{3} q}{(2 \pi)^{3}} \mathcal{M}_{R}(q) \mathcal{M}_{R}\left(\left|\boldsymbol{k}_{1}-\boldsymbol{q}\right|\right) P_{\phi}(q)+(2 \text { cyclic }) \\
& \quad+4 \mathcal{M}_{R}\left(k_{2}\right) \mathcal{M}_{R}\left(k_{3}\right) P_{\phi}\left(k_{2}\right) P_{\phi}\left(k_{3}\right) \\
& \quad \times \int \frac{d^{3} q}{(2 \pi)^{3}} \mathcal{M}_{R}(q) \mathcal{M}_{R}\left(\left|\boldsymbol{k}_{1}-\boldsymbol{q}\right|\right) \\
& \left.\quad \times\left[P_{\phi}\left(\left|\boldsymbol{k}_{2}+\boldsymbol{q}\right|\right)+P_{\phi}\left(\left|\boldsymbol{k}_{3}+\boldsymbol{q}\right|\right)\right]+(2 \text { cyclic })\right], \\
& \quad \times \int \frac{d^{3} q}{(2 \pi)^{3}} \mathcal{M}_{R}(q) \mathcal{M}_{R}\left(\left|\boldsymbol{k}_{1}-\boldsymbol{q}\right|\right) P_{\phi}(q) P_{\phi}\left(\left|\boldsymbol{k}_{1}-\boldsymbol{q}\right|\right) \\
& \quad+(2 \mathrm{cyclic})+12 \mathcal{M}_{R}\left(k_{2}\right) \mathcal{M}_{R}\left(k_{3}\right) P_{\phi}\left(k_{2}\right) P_{\phi}\left(k_{3}\right) \\
& \left.\quad \times \int \frac{d^{3} q}{(2 \pi)^{3}} \mathcal{M}_{R}(q) \mathcal{M}_{R}\left(\left|\boldsymbol{k}_{1}-\boldsymbol{q}\right|\right) P_{\phi}(q)+(2 \text { cyclic })\right] .
\end{aligned}
$$

We find that the first three cyclic terms in Equation (29) are parametrically small on large scales and may be ignored for $k \lesssim 0.1 h \mathrm{Mpc}^{-1}$. Therefore, one may just calculate the last two cyclic terms

$$
\begin{aligned}
& B_{f_{\mathrm{NL}}^{2}}^{n G}\left(k_{1}, k_{2}, k_{3}\right) \approx \frac{1}{2 \sigma_{R}^{2}}\left[8 \mathcal{M}_{R}\left(k_{2}\right) \mathcal{M}_{R}\left(k_{3}\right) P_{\phi}\left(k_{1}\right)\right. \\
& \quad \times\left[P_{\phi}\left(k_{2}\right)+P_{\phi}\left(k_{3}\right)\right] \int \frac{d^{3} q}{(2 \pi)^{3}} \mathcal{M}_{R}(q) \mathcal{M}_{R}\left(\left|\boldsymbol{k}_{1}-\boldsymbol{q}\right|\right) \\
& \quad \times P_{\phi}(q)+(2 \text { cyclic })+4 \mathcal{M}_{R}\left(k_{2}\right) \mathcal{M}_{R}\left(k_{3}\right) P_{\phi}\left(k_{2}\right) P_{\phi}\left(k_{3}\right) \\
& \quad \times \int \frac{d^{3} q}{(2 \pi)^{3}} \mathcal{M}_{R}(q) \mathcal{M}_{R}\left(\left|\boldsymbol{k}_{1}-\boldsymbol{q}\right|\right) \\
& \left.\quad \times\left[P_{\phi}\left(\left|\boldsymbol{k}_{2}+\boldsymbol{q}\right|\right)+P_{\phi}\left(\left|\boldsymbol{k}_{3}+\boldsymbol{q}\right|\right)\right]+(2 \text { cyclic })\right] .
\end{aligned}
$$

Next, the second term of Equation (27) contains a crosscorrelation between the non-linearly evolved density field $\left(\delta^{(2)} \sim F_{2}^{(s)}\left[\delta^{(1)}\right]^{2}\right)$ and the primordial bispectrum, and thus it is linearly proportional to $f_{\mathrm{NL}}$ and $F_{2}^{(s)}$. We present the explicit functional form of $T_{R}^{1112}$ as well as the full expression of the second term of Equation (27) in Appendix A. Here, we only show the final result. We write it as

$$
\begin{aligned}
& \frac{1}{2 \sigma_{R}^{2}} \int \frac{d^{3} q}{(2 \pi)^{3}} T_{R}^{(2)}\left(\boldsymbol{q}, \boldsymbol{k}_{1}-\boldsymbol{q}, \boldsymbol{k}_{2}, \boldsymbol{k}_{3}\right)+(2 \text { cyclic }) \\
& =f_{\mathrm{NL}}\left[B_{m}^{n G}\left(k_{1}, k_{2}, k_{3}\right)+B_{f_{\mathrm{NL}}^{n G 1}}^{n G}\left(k_{1}, k_{2}, k_{3}\right)\right. \\
& \left.\quad+4 B_{f_{\mathrm{NL}}^{n G 0}}^{n G 0}\left(k_{1}, k_{2}, k_{3}\right)\left\{\mathcal{G}_{R}\left(k_{1}\right)+\mathcal{G}_{R}\left(k_{2}\right)+\mathcal{G}_{R}\left(k_{3}\right)\right\}\right]
\end{aligned}
$$

where

$$
\begin{aligned}
& B_{m}^{n G}\left(k_{1}, k_{2}, k_{3}\right) \equiv 4 \tilde{W}_{R}\left(k_{1}\right) \tilde{W}_{R}\left(k_{2}\right) \tilde{W}_{R}\left(k_{3}\right) \\
& \quad \times\left\{\left[\frac{\mathcal{F}_{R}\left(k_{1}\right)}{\mathcal{M}_{R}\left(k_{1}\right)}+\frac{\mathcal{F}_{R}\left(k_{2}\right)}{\mathcal{M}_{R}\left(k_{2}\right)}\right] P_{m}\left(k_{1}\right) P_{m}\left(k_{2}\right) F_{2}^{(s)}\left(\boldsymbol{k}_{1}, \boldsymbol{k}_{2}\right)\right. \\
& \quad+(2 \text { cyclic })\},
\end{aligned}
$$

$$
\begin{aligned}
& B_{f_{\mathrm{NL}}}^{n G 1}\left(k_{1}, k_{2}, k_{3}\right) \equiv \frac{1}{2 \sigma_{R}^{2}}\left[8 \tilde{W}_{R}\left(k_{2}\right) \tilde{W}_{R}\left(k_{3}\right) \mathcal{M}\left(k_{3}\right) P_{m}\left(k_{2}\right)\right. \\
& \quad \times \int \frac{d^{3} q}{(2 \pi)^{3}} \tilde{W}_{R}\left(\left|\boldsymbol{k}_{1}-\boldsymbol{q}\right|\right) \tilde{W}_{R}(q) \mathcal{M}\left(\left|\boldsymbol{k}_{1}-\boldsymbol{q}\right|\right) \mathcal{M}\left(\left|\boldsymbol{k}_{2}+\boldsymbol{q}\right|\right) \\
& \quad \times F_{2}^{(s)}\left(-\boldsymbol{k}_{2}, \boldsymbol{k}_{2}+\boldsymbol{q}\right)\left\{P_{\phi}\left(k_{3}\right) P_{\phi}\left(\left|\boldsymbol{k}_{1}-\boldsymbol{q}\right|\right)\right. \\
& \left.\quad+P_{\phi}\left(k_{3}\right) P_{\phi}\left(\left|\boldsymbol{k}_{2}+\boldsymbol{q}\right|\right)+P_{\phi}\left(\left|\boldsymbol{k}_{1}-\boldsymbol{q}\right|\right) P_{\phi}\left(\left|\boldsymbol{k}_{2}+\boldsymbol{q}\right|\right)\right\} \\
& \quad+(5 \text { permutation })+8 \tilde{W}_{R}\left(k_{2}\right) \tilde{W}_{R}\left(k_{3}\right) \mathcal{M}\left(k_{3}\right) \\
& \quad \times \int \frac{d^{3} q}{(2 \pi)^{3}} \tilde{W}_{R}\left(\left|\boldsymbol{k}_{1}-\boldsymbol{q}\right|\right) \tilde{W}_{R}(q) \mathcal{M}\left(\left|\boldsymbol{k}_{1}-\boldsymbol{q}\right|\right) \\
& \quad \times \mathcal{M}\left(\left|\boldsymbol{k}_{2}+\boldsymbol{q}\right|\right) P_{m}(q) F_{2}^{(s)}\left(-\boldsymbol{q}, \boldsymbol{k}_{2}+\boldsymbol{q}\right) \\
& \quad \times\left\{P_{\phi}\left(\left|\boldsymbol{k}_{1}-\boldsymbol{q}\right|\right) P_{\phi}\left(k_{3}\right)+P_{\phi}\left(\left|\boldsymbol{k}_{1}-\boldsymbol{q}\right|\right) P_{\phi}\left(\left|\boldsymbol{k}_{2}+\boldsymbol{q}\right|\right)\right. \\
& \left.\quad+P_{\phi}\left(k_{3}\right) P_{\phi}\left(\left|\boldsymbol{k}_{2}+\boldsymbol{q}\right|\right)\right\}+(5 \text { permutation }) \\
& \quad+8\left(\tilde{W}_{R}\left(k_{2}\right) \tilde{W}_{R}\left(k_{3}\right)\right)^{2} P_{m}\left(k_{3}\right) \mathcal{M}\left(k_{2}\right) \\
& \quad \times \int \frac{d^{3} p}{(2 \pi)^{3}} \mathcal{M}(p) \mathcal{M}\left(\left|\boldsymbol{k}_{2}-\boldsymbol{p}\right|\right) P_{\phi}(p)\left\{P_{\phi}\left(\left|\boldsymbol{k}_{2}-\boldsymbol{p}\right|\right)\right. \\
& \left.\left.\quad+2 P_{\phi}\left(k_{2}\right)\right\} F_{2}^{(s)}\left(\boldsymbol{p}, \boldsymbol{k}_{2}-\boldsymbol{p}\right)+(5 \text { permutation })\right] .
\end{aligned}
$$

Here, $\mathcal{M}(k) \equiv \mathcal{M}_{R}(k) / \tilde{W}_{R}(k)$.

In the above equations, we have defined two functions, $\mathcal{F}_{R}(k)$ and $\mathcal{G}_{R}(k)$, which are given by

$$
\begin{aligned}
& \mathcal{F}_{R}(k) \equiv \frac{1}{2 \sigma_{R}^{2}} \int \frac{d^{3} q}{(2 \pi)^{3}} P_{\phi}(q) \mathcal{M}_{R}(q) \mathcal{M}_{R}(|\boldsymbol{k}-\boldsymbol{q}|) \\
& \times\left[\frac{P_{\phi}(|\boldsymbol{k}-\boldsymbol{q}|)}{P_{\phi}(k)}+2\right], \\
& \mathcal{G}_{R}(k) \equiv \frac{1}{2 \sigma_{R}^{2}} \int \frac{d^{3} q}{(2 \pi)^{3}} \frac{\tilde{W}_{R}(q) \tilde{W}_{R}(|\boldsymbol{k}-\boldsymbol{q}|)}{\tilde{W}_{R}(k)} \\
& \times P_{m}(q) F_{2}^{(s)}(\boldsymbol{k},-\boldsymbol{q}) .
\end{aligned}
$$

As shown in Figures 5 and 6, both $\mathcal{F}_{R}(k)$ and $\mathcal{G}_{R}(k)$ are almost constant on large scales. If we do not have a smoothing, i.e., $R \rightarrow 0$, the large scale asymptotic value of $\mathcal{G}_{R}(k)$ is $17 / 42$. However, the presence of filter changes this asymptotic value. As $k \rightarrow 0$,

$$
\mathcal{G}_{R}(k) \rightarrow \frac{13}{84}+\frac{1}{4 \sigma_{R}^{2}} \int \frac{d^{3} q}{(2 \pi)^{3}} \tilde{W}_{R}(q) P_{m}(q) \frac{\sin (q R)}{q R},
$$

whose value depends on the smoothing scale, $R$, as shown in Figure 7.

Let us study the structure of each term in Equation (32). The first piece is $B_{m}^{n G}$. On very large scales, where $\tilde{W}_{R}(k) \rightarrow 1$ and $\mathcal{F}_{R}(k) \rightarrow 1, B_{m}^{n G}$ becomes a product of the usual matter bispectrum for Gaussian initial conditions, $B_{m}^{G}$, and the scale dependent bias shown in Equation (4), as

$$
\frac{2 f_{\mathrm{NL}} \mathcal{F}_{R}(k)}{\mathcal{M}_{R}(k)}=\frac{3 f_{\mathrm{NL}} H_{0}^{2} \Omega_{m}}{k^{2} T(k)} \frac{\mathcal{F}_{R}(k)}{\tilde{W}_{R}(k)} \rightarrow \frac{3 f_{\mathrm{NL}} H_{0}^{2} \Omega_{m}}{k^{2} T(k)},
$$

as $k \rightarrow 0$. Therefore, we can interpret this term as a scale dependent bias multiplying the usual matter bispectrum for Gaussian initial conditions; however, this behavior is not generic - in fact, 


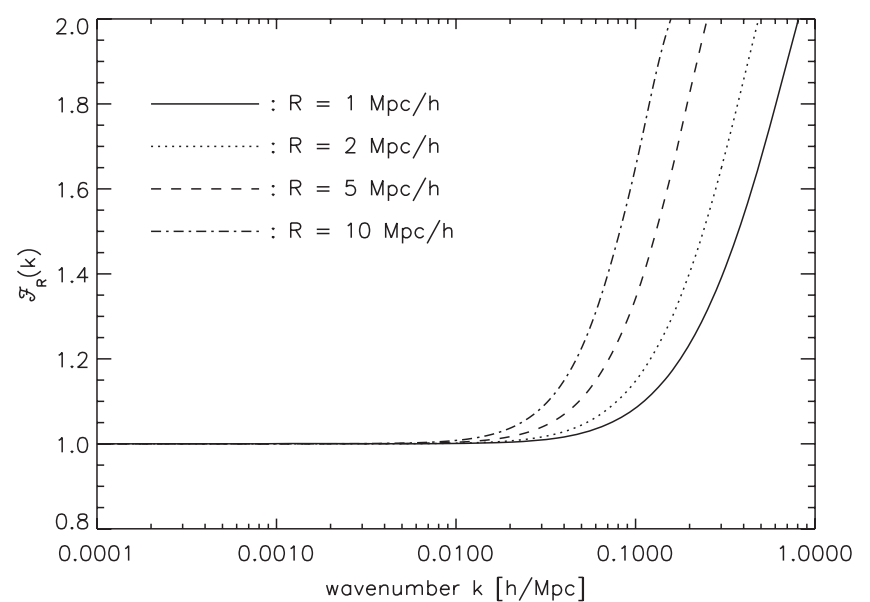

Figure 5. Shape of the function, $\mathcal{F}_{R}(k)$, defined in Equation (35). We show $\mathcal{F}_{R}(k)$ for four different smoothing lengths: $R=1,2,5,10 \mathrm{Mpc} h^{-1}$.

the other terms cannot be expressed in terms of products of the scale-dependent bias and the results in the continuous limit, Equation (1)

The next piece is $B_{f_{\mathrm{NL}}}^{n G 1}\left(k_{1}, k_{2}, k_{3}\right)$. By numerically calculating Equation (34), we find that the terms that contain $F_{2}^{(s)}(\boldsymbol{q}, \boldsymbol{k}-\boldsymbol{q})$ are parametrically small on large scales, and that the dominant contributions come from the first permutation terms. Therefore, we approximate Equation (34) on large scale $\left(k \lesssim 0.1 \mathrm{~h} \mathrm{Mpc}^{-1}\right)$ as

$$
\begin{aligned}
& B_{f_{\mathrm{NL}}}^{n G 1}\left(k_{1}, k_{2}, k_{3}\right) \approx \frac{1}{2 \sigma_{R}^{2}}\left[8 \tilde{W}_{R}\left(k_{2}\right) \tilde{W}_{R}\left(k_{3}\right) P_{m}\left(k_{2}\right) \mathcal{M}\left(k_{3}\right) P_{\phi}\left(k_{3}\right)\right. \\
& \quad \times \int \frac{d^{3} q}{(2 \pi)^{3}} \tilde{W}_{R}\left(\left|\boldsymbol{k}_{1}-\boldsymbol{q}\right|\right) \tilde{W}_{R}(q) \mathcal{M}\left(\left|\boldsymbol{k}_{1}-\boldsymbol{q}\right|\right) \mathcal{M}\left(\left|\boldsymbol{k}_{2}+\boldsymbol{q}\right|\right) \\
& \quad \times\left[P_{\phi}\left(\left|\boldsymbol{k}_{2}+\boldsymbol{q}\right|\right)+P_{\phi}\left(\left|\boldsymbol{k}_{1}-\boldsymbol{q}\right|\right)\right] F_{2}^{(s)}\left(-\boldsymbol{k}_{2}, \boldsymbol{k}_{2}+\boldsymbol{q}\right) \\
& \quad+(5 \text { permutation })] .
\end{aligned}
$$

How about the last term of Equation (32), $4 B_{f_{\mathrm{NL}}}^{n G 0}\left(k_{1}, k_{2}, k_{3}\right)$ $\left\{\mathcal{G}_{R}\left(k_{1}\right)+\mathcal{G}_{R}\left(k_{2}\right)+\mathcal{G}_{R}\left(k_{3}\right)\right\}$ ? As $\mathcal{G}_{R}(k) \rightarrow$ constant on large scales (Figure 6), this piece becomes $B_{f_{\mathrm{NL}}}^{n G 0}$ multiplied by a constant factor whose exact value depends on the smoothing scale, $R$ (Figure 7).

In summary, we have derived the new terms in the galaxy bispectrum, which arise from the integration of the matter trispectrum. While we find one term, $B_{m}^{n G}$, includes the scaledependent bias which appears on the galaxy power spectrum, we also find that there are more terms contributing to the galaxy bispectrum.

Equation (28) along with Equations (29)-(31), and Equation (32) along with Equations (33), (34), and (39) are the second main results of this paper. In the next sections, we shall present the detailed assessment of the new terms we have derived in this section.

\subsubsection{Shape Dependence}

Let us consider the shape dependence. First of all, the last term of Equation (32) has the same shape dependence as $B_{f_{N} G 0}$, as $\mathcal{G}_{R}(k)$ is almost constant on large scale. Thus, it peaks at the squeezed configurations as $B_{f \mathrm{NL}}^{n G 0}$ does. How about the shape dependence of the other terms?

All terms in Equations (30), (31), and (39) have $P_{\phi}\left(k_{i}\right)$ outside of the integral, and Equation (33) contains $1 / \mathcal{M}_{R}(k) \propto k^{-2}$,

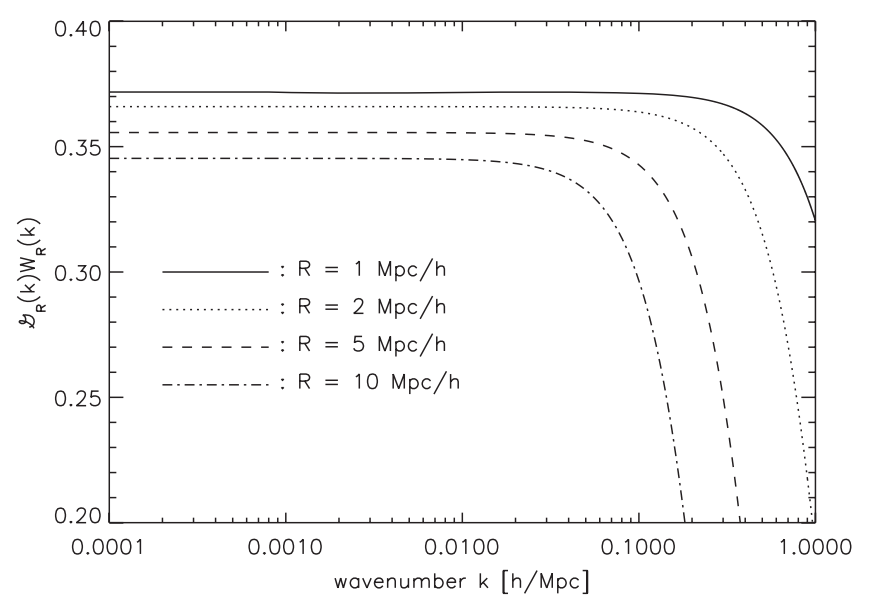

Figure 6. Shape of the function, $\mathcal{G}_{R}(k)$, defined in Equation (35). We show $\mathcal{G}_{R}(k)$ for four different smoothing lengths: $R=1,2,5,10 \mathrm{Mpc} h^{-1}$.

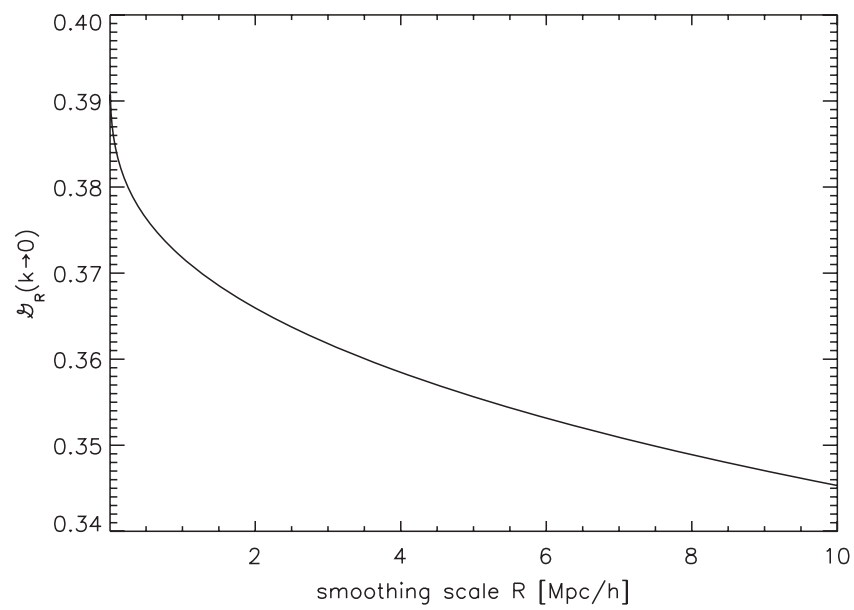

Figure 7. Large-scale asymptotic value of $\mathcal{G}_{R}(k)$ as a function of the smoothing scale $R$. The value for $R=1\left[\mathrm{Mpc} h^{-1}\right]$, which is used for generating Figures $10-14$, is 0.3718 .

which suggests that all of $B_{g_{\mathrm{NL}}}^{n G}, B_{f_{\mathrm{NL}}^{2}}^{n G}, B_{f_{\mathrm{NL}}}^{n G 1}$, and $B_{m}^{n G}$ peak at the squeezed configurations. For sufficiently large scales in which $T(k) \approx 1$, and for a scale-invariant spectrum $\left(P_{\phi}(k) \propto 1 / k^{3}\right)$, we may write down Equations (33), (39), (31), and (30), as

$$
\begin{aligned}
B_{m}^{n G}\left(k_{1}, k_{2}, k_{3}\right) \propto & \left(\frac{k_{2}}{k_{1}}+\frac{k_{1}}{k_{2}}\right) \\
& \times\left[\frac{5}{7}+\frac{\boldsymbol{k}_{1} \cdot \boldsymbol{k}_{2}}{2 k_{1} k_{2}}\left(\frac{k_{1}}{k_{2}}+\frac{k_{2}}{k_{1}}\right)+\frac{2}{7}\left(\frac{\boldsymbol{k}_{1} \cdot \boldsymbol{k}_{2}}{k_{1} k_{2}}\right)^{2}\right] \\
& +(2 \text { cyclic }),
\end{aligned}
$$

$$
\begin{aligned}
B_{f_{\mathrm{NL}}}^{n G 1}\left(k_{1}, k_{2}, k_{3}\right) \propto & \frac{k_{2}}{k_{3}} \int \frac{d^{3} q}{q} q^{3}\left(\frac{\left|\boldsymbol{k}_{1}-\boldsymbol{q}\right|^{2}}{\left|\boldsymbol{k}_{2}+\boldsymbol{q}\right|}+\frac{\left|\boldsymbol{k}_{2}+\boldsymbol{q}\right|^{2}}{\left|\boldsymbol{k}_{1}-\boldsymbol{q}\right|}\right) \\
& \times \tilde{W}_{R}\left(\left|\boldsymbol{k}_{1}-\boldsymbol{q}\right|\right) \tilde{W}_{R}(q) T\left(\left|\boldsymbol{k}_{1}-\boldsymbol{q}\right|\right) \\
& \times T\left(\left|\boldsymbol{k}_{2}+\boldsymbol{q}\right|\right) F_{2}^{(s)}\left(-\boldsymbol{k}_{2}, \boldsymbol{k}_{2}+\boldsymbol{q}\right) \\
& +(5 \text { permutation }),
\end{aligned}
$$

$$
\begin{aligned}
B_{f_{\mathrm{NL}}^{2}}^{n G}\left(k_{1}, k_{2}, k_{3}\right) \propto & \frac{4}{k_{2} k_{3}}\left[\frac{2\left(k_{2}^{3}+k_{3}^{3}\right)}{k_{1}^{3}} \int \frac{d^{3} q}{q}\left|\boldsymbol{k}_{1}-\boldsymbol{q}\right|^{2} T(q)\right. \\
& \times \tilde{W}_{R}(q) T\left(\left|\boldsymbol{k}_{1}-\boldsymbol{q}\right|\right) \tilde{W}_{R}\left(\left|\boldsymbol{k}_{1}-\boldsymbol{q}\right|\right)
\end{aligned}
$$



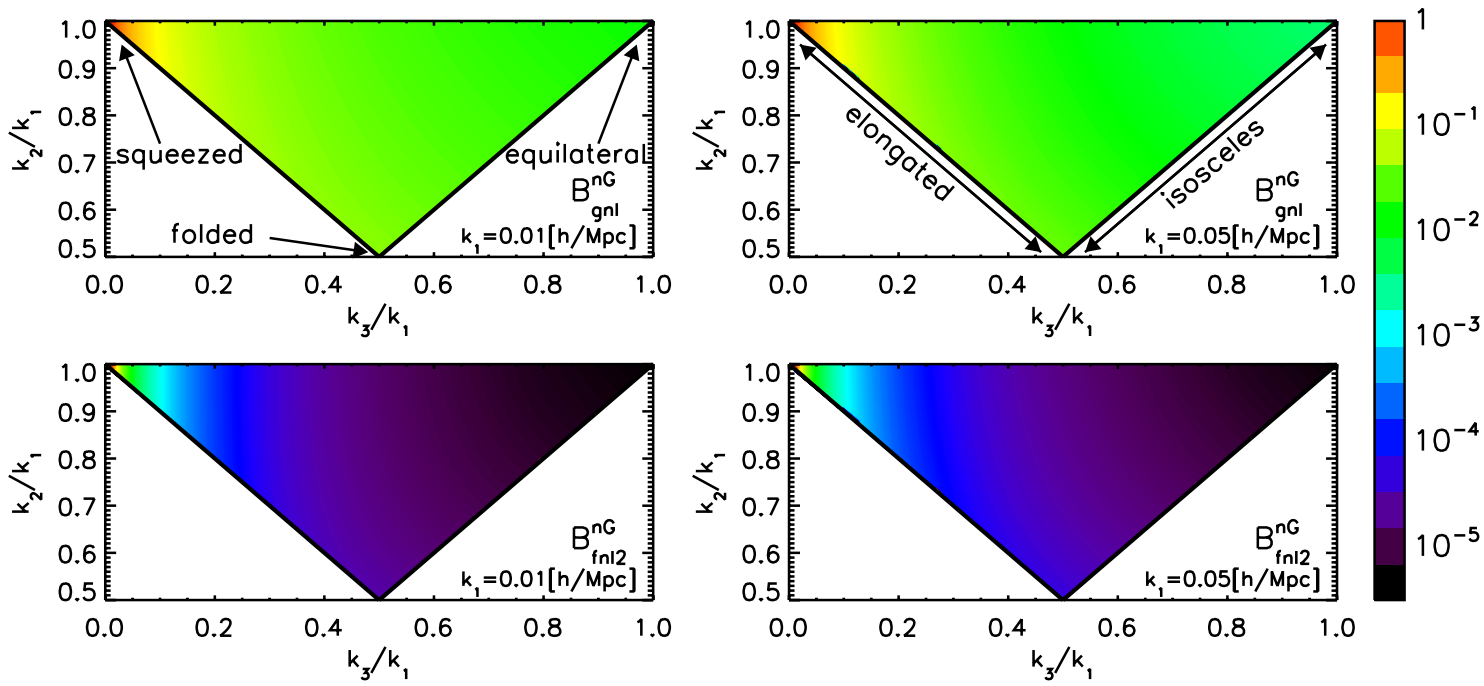

Figure 8. Same as Figure 2, but for (Top) the $g_{\mathrm{NL}}$ term (Equation (30)), and (Bottom) the $f_{\mathrm{NL}}^{2}$ term (Equation (31)). Note that the non-Gaussian terms diverge in the exact squeezed limit, $k_{3} \rightarrow 0$; thus, we show these terms normalized to be unity at $k_{3} / k_{1}=10^{-2}$. In order to facilitate the comparison better, we draw the dotted contour for top panels.

(A color version of this figure is available in the online journal.)

$$
\begin{aligned}
& +\int d^{3} q q^{2}\left|\boldsymbol{k}_{1}-\boldsymbol{q}\right|^{2}\left(\frac{1}{\left|\boldsymbol{k}_{2}+\boldsymbol{q}\right|^{3}}+\frac{1}{\left|\boldsymbol{k}_{3}+\boldsymbol{q}\right|^{3}}\right) \\
& \times T(q) \tilde{W}_{R}(q) T\left(\left|\boldsymbol{k}_{1}-\boldsymbol{q}\right|\right) \tilde{W}_{R}\left(\left|\boldsymbol{k}_{1}-\boldsymbol{q}\right|\right) \\
& +(2 \text { cyclic }),
\end{aligned}
$$

$$
\begin{aligned}
B_{g_{\mathrm{NL}}}^{n G}\left(k_{1}, k_{2}, k_{3}\right) \propto & \frac{6}{k_{2} k_{3}}\left[\left(k_{2}^{3}+k_{3}^{3}\right) \int \frac{d^{3} q}{q} \frac{1}{\left|\boldsymbol{k}_{1}-\boldsymbol{q}\right|}\right. \\
& \times T(q) \tilde{W}_{R}(q) T\left(\left|\boldsymbol{k}_{1}-\boldsymbol{q}\right|\right) \tilde{W}_{R}\left(\left|\boldsymbol{k}_{1}-\boldsymbol{q}\right|\right) \\
& +2 \int \frac{d^{3} q}{q}\left|\boldsymbol{k}_{1}-\boldsymbol{q}\right|^{2} T(q) \tilde{W}_{R}(q) T\left(\left|\boldsymbol{k}_{1}-\boldsymbol{q}\right|\right) \\
& \left.\times \tilde{W}_{R}\left(\left|\boldsymbol{k}_{1}-\boldsymbol{q}\right|\right)\right]+(2 \text { cyclic }),
\end{aligned}
$$

respectively. For a given $k_{1}$, all of these terms have the largest signals when $k_{3}$ is small, i.e., the squeezed configurations. Note that we do not use the exact scale-invariant spectrum for the numerical calculation, but use the WMAP five-year best-fitting value reported in Table 1 ("WMAP+BAO+SN") of Komatsu et al. (2009).

The top-left and bottom-left panels of Figure 4 show $B_{m}^{n G}$ and $B_{f_{\mathrm{NL}}}^{n G 1}$ as a function of $k_{2} / k_{1}$ and $k_{3} / k_{1}$, respectively, for $k_{1}=0.01 h \mathrm{Mpc}^{-1}$. The top-right and bottom-right panels of Figure 4 show the same quantities for $k_{1}=0.05 h \mathrm{Mpc}^{-1}$. We also show $B_{g_{\mathrm{NL}}}^{n G}$ and $B_{f_{\mathrm{NL}}^{2}}^{n G}$ in the top-left and bottom-left panels of Figure 8 for $k_{1}=0.01 h \mathrm{Mpc}^{-1}$, and top-right and bottom-right for for $k_{1}=0.05 h \mathrm{Mpc}^{-1}$. In all cases, we find that $B_{m}^{n G}, B_{f_{\mathrm{NL}}}^{n G 1}$, $B_{g_{\mathrm{NL}}}^{n G}$ and $B_{f_{\mathrm{NL}}^{2}}^{n G}$ peak at the squeezed configurations, as expected from the above argument.

We find that the shape dependence of $B_{f_{\mathrm{NL}}}^{n G 0}$ and that of $B_{f_{\mathrm{NL}}}^{n G 1}$, $B_{g_{\mathrm{NL}}}^{n G}$ are quite similar, whereas that of $B_{m}^{n G}$ is higher toward the elongated triangles, and that of $B_{f_{\mathrm{NL}}^{2}}^{n G}$ is more sharply peaked at the squeezed configuration.

We can understand this behavior analytically as follows. In order to simplify the analysis, we consider a scale-invariant curvature power spectrum, $P_{\phi}=P_{\phi 0} / k^{3}$, on large scales where
Equations (41), (40), (42), and (43) are valid. On such a large scale, $\mathcal{M}_{R}(k)$ can be approximated as $\mathcal{M}_{R}(k) \simeq 2 k^{2} /\left(3 H_{0}^{2} \Omega_{m}\right) \equiv \mathcal{M}_{0} k^{2}$, where $M_{0} \simeq 2.16 \times$ $10^{7}\left(0.277 / \Omega_{m}\right)[\mathrm{Mpc} / h]^{2}$ is a constant. We focus on the squeezed triangle, $k_{1}=k_{2}=\alpha k_{3} \equiv k(\alpha \gg 1)$, where the signals of all the primordial non-Gaussianity terms are maximized. The triangles in this configuration lie on the upper side of the triangular region of $\left(k_{3} / k_{1}, k_{2} / k_{1}\right)$ plane in Figures 4 and 8 , and the triangle approaches the exact squeezed limit as $\alpha \rightarrow \infty$. With this parametrization, we compare the dominant contributions of each of these primordial non-Gaussianity terms.

First, we shall analyze the terms proportional to $f_{\mathrm{NL}}: B_{f_{\mathrm{NL}}}^{n G 0}$, $B_{m}^{n G}$, and $B_{f_{\mathrm{NL}}}^{n G 1}$. The largest contribution to $B_{f_{\mathrm{NL}}}^{n G 0}$ in the squeezed configurations occurs when $k_{3}$ is in the denominator

$$
\begin{aligned}
B_{f_{\mathrm{NL}}}^{n G 0} & =2 \mathcal{M}_{0}^{3} P_{\phi 0}^{2}\left(\frac{k_{1}^{2}}{k_{2} k_{3}}+\frac{k_{2}^{2}}{k_{3} k_{1}}+\frac{k_{3}^{2}}{k_{1} k_{2}}\right) \\
& \simeq 2 \mathcal{M}_{0}^{3} P_{\phi 0}^{2}\left(\frac{k_{1}^{2}}{k_{2} k_{3}}+\frac{k_{2}^{2}}{k_{3} k_{1}}\right) \\
& =4 \alpha \mathcal{M}_{0}^{3} P_{\phi 0}^{2} .
\end{aligned}
$$

To compute $B_{m}^{n G}$, which contains $F_{2}^{(s)}$, we note that, in the squeezed limit, the angular cosines between two wave vectors are $\boldsymbol{k}_{1} \cdot \boldsymbol{k}_{2} /\left(k_{1} k_{2}\right)=-1+1 / \alpha^{2} \simeq-1$ and $\boldsymbol{k}_{2} \cdot \boldsymbol{k}_{3} /\left(k_{2} k_{3}\right)=$ $\boldsymbol{k}_{1} \cdot \boldsymbol{k}_{3} /\left(k_{1} k_{3}\right)=-1 /(2 \alpha)$. We thus find

$$
\begin{aligned}
B_{m}^{n G} & =8 \mathcal{M}_{0}^{3} P_{\phi 0}^{2}\left(\alpha+\frac{1}{\alpha}\right)\left[\frac{5}{7}-\frac{1}{4 \alpha}\left(\alpha+\frac{1}{\alpha}\right)+\frac{1}{14 \alpha^{2}}\right] \\
& \simeq \frac{26}{7} \alpha \mathcal{M}_{0}^{3} P_{\phi 0}^{2} .
\end{aligned}
$$

The detailed analysis for $B_{f_{\mathrm{NL}}}^{n G 1}$ is more complicated, as Equation (39) involves a non-trivial integration. We simplify the situation by only analyzing the dominant term, which can be written as

$$
\begin{aligned}
B_{f_{\mathrm{NL}}}^{n G 1} & \approx 8 \mathcal{M}_{0}^{3} P_{\phi 0}^{2}\left[\frac{k_{2}}{k_{3}} \mathcal{H}\left(\boldsymbol{k}_{1}, \boldsymbol{k}_{2}\right)+\frac{k_{1}}{k_{3}} \mathcal{H}\left(\boldsymbol{k}_{2}, \boldsymbol{k}_{1}\right)\right] \\
& =8 \alpha \mathcal{M}_{0}^{3} P_{\phi 0}^{2}\left[\mathcal{H}\left(\boldsymbol{k}_{1}, \boldsymbol{k}_{2}\right)+\mathcal{H}\left(\boldsymbol{k}_{2}, \boldsymbol{k}_{1}\right)\right],
\end{aligned}
$$




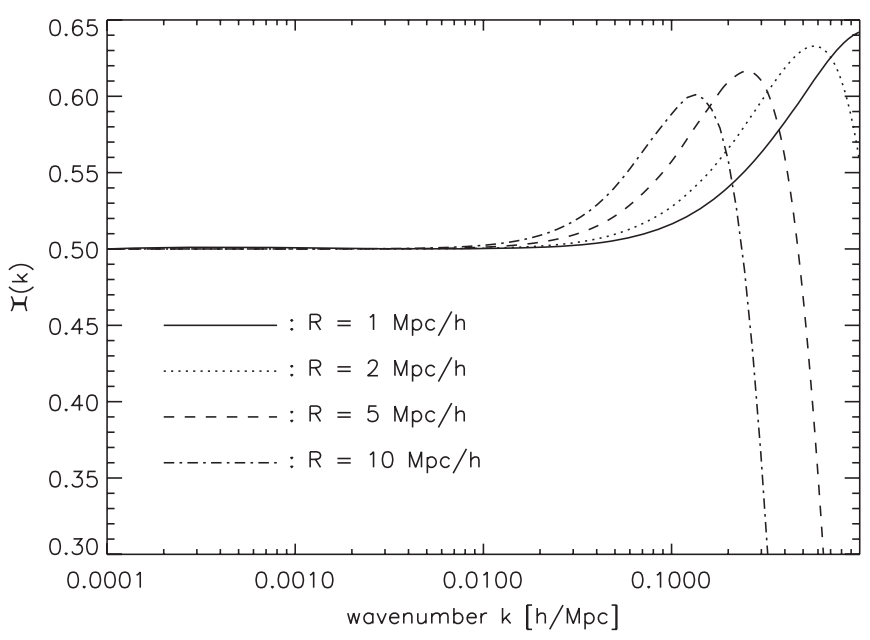

Figure 9. Shape of the integration that appears in the dominant term of $B_{f_{\mathrm{NL}}^{2}}^{n G}$ and $B_{g_{\mathrm{NL}}}^{n G}$, Equation (48). We use four different smoothing scales: $R=1,2,5$, and $10 \mathrm{Mpc} h^{-1}$.

where $\mathcal{H}\left(\boldsymbol{k}_{1}, \boldsymbol{k}_{2}\right)$ is the integration that appears in Equation (39) including $1 /\left(2 \sigma_{R}^{2}\right)$ pre-factor. Note that this integration depends only on the magnitudes of two vectors and the angle between them; thus, for the squeezed configuration we are interested in here, $\mathcal{H}\left(\boldsymbol{k}_{2}, \boldsymbol{k}_{1}\right)$ depends only weakly on $\alpha$-they depend on $\alpha$ only through the inner product of $\boldsymbol{k}_{1} \cdot \boldsymbol{k}_{2}=k^{2}\left(-1+1 / \alpha^{2}\right)$.

Second, we analyze $B_{g_{\mathrm{NL}}}^{n G}$. We find that the first cyclic terms in Equation (30) are small in the squeezed limit, and the dominant contribution to $B_{g_{\mathrm{NL}}}^{n G}$ is given by

$$
\begin{aligned}
B_{g_{\mathrm{NL}}}^{n G} & =12 \mathcal{M}_{0}^{2} P_{\phi 0}^{2}\left[\frac{\mathcal{I}\left(k_{1}\right)}{k_{2} k_{3}}+\frac{\mathcal{I}\left(k_{2}\right)}{k_{3} k_{1}}+\frac{\mathcal{I}\left(k_{3}\right)}{k_{1} k_{2}}\right] \\
& \simeq 12 \mathcal{M}_{0}^{2} P_{\phi 0}^{2}\left[\frac{\mathcal{I}\left(k_{1}\right)}{k_{2} k_{3}}+\frac{\mathcal{I}\left(k_{2}\right)}{k_{3} k_{1}}\right],
\end{aligned}
$$

where we have defined

$$
\mathcal{I}(k) \equiv \frac{1}{2 \sigma_{R}^{2}} \int \frac{d^{3} q}{(2 \pi)^{3}} \mathcal{M}_{R}(q) \mathcal{M}_{R}(|\boldsymbol{k}-\boldsymbol{q}|) P_{\phi}(q) .
$$

We find that $\mathcal{I}(k) \simeq 0.5$ and is almost independent of $k$ on large scales (e.g., $k \lesssim 0.03 h \mathrm{Mpc}^{-1}$ for $R=1.0 \mathrm{Mpc} h^{-1}$; see Figure 9). Therefore, by writing $\mathcal{I}(k)=\mathcal{I}_{0}$, we obtain

$$
B_{g_{\mathrm{NL}}}^{n G} \simeq 24 \alpha \mathcal{M}_{0}^{2} P_{\phi 0}^{2} \frac{\mathcal{I}_{0}}{k^{2}}
$$

These results show that all the terms we have analyzed analytically so far, $B_{f_{\mathrm{NL}}}^{n G 0}, B_{m}^{n G}, B_{f_{\mathrm{NL}}}^{n G 1}$, and $B_{g_{\mathrm{NL}}}^{n G}$, have the same shape (i.e., $\alpha$ ) dependence in the squeezed configurations: they both increase linearly as $\alpha$ increases. This explains the shape dependence computed from the full numerical calculations presented in Figure 4 and the top panels of Figure 8.

Finally, we analyze $B_{f_{\mathrm{NL}}^{2}}^{n}$. We find that the second cyclic terms in Equation (31) are small in the squeezed configurations. The dominant terms are

$$
\begin{aligned}
B_{f_{\mathrm{NL}}^{2}}^{n G}= & 8 \mathcal{M}_{0}^{2} P_{\phi 0}^{2} \\
& \times\left[\frac{k_{2}^{3}+k_{3}^{3}}{k_{2} k_{3} k_{1}^{3}} \mathcal{I}\left(k_{1}\right)+\frac{k_{3}^{3}+k_{1}^{3}}{k_{3} k_{1} k_{2}^{3}} \mathcal{I}\left(k_{2}\right)+\frac{k_{1}^{3}+k_{2}^{3}}{k_{1} k_{2} k_{3}^{3}} \mathcal{I}\left(k_{3}\right)\right]
\end{aligned}
$$

$$
\begin{aligned}
& \simeq 8 \mathcal{M}_{0}^{2} P_{\phi 0}^{2} \frac{k_{1}^{3}+k_{2}^{3}}{k_{1} k_{2} k_{3}^{3}} \mathcal{I}\left(k_{3}\right) \\
& \simeq 16 \alpha^{3} \mathcal{M}_{0}^{2} P_{\phi 0}^{2} \frac{\mathcal{I}_{0}}{k^{2}}
\end{aligned}
$$

Therefore, $B_{f_{\mathrm{NL}}^{2}}^{n G}$ increases more sharply as it approaches the squeezed limit, $B_{f_{\mathrm{NL}}^{2}}^{n G} \propto \alpha^{3}$.

This sharp increase of $B_{f_{\mathrm{NL}}^{2}}^{n G}$ relative to the other terms, and that there are many new terms that are of the same order of magnitude as $B_{f_{\mathrm{NL}}}^{n G 0}$, imply that the formula derived by Sefusatti \& Komatsu (2007) may not be valid in the squeezed configuration, where $B_{f_{\mathrm{NL}}^{2}}^{n G}$ may dominate over $B_{f_{\mathrm{NL}}}^{n G}$. This is particularly important because it is the squeezed configuration that gives the largest signal from the primordial non-Gaussianity. We shall study this point in more detail in the next section.

A careful inspection of Equation (42) shows that the second term within the square bracket diverges when $\boldsymbol{k}_{2}+\boldsymbol{q}=0$ or $\boldsymbol{k}_{3}+\boldsymbol{q}=0$. This is due to the fact that $P_{\phi}(k) \propto 1 / k^{4-n_{s}}$ and thus $P_{\phi}(k)$ diverges as $k \rightarrow 0$ for $n_{s}<4$. To avoid the divergence we set $P_{\phi}(k)=0$ at $k \leqslant k_{\min }$, and use $k_{\min }=10^{-6} h \mathrm{Mpc}^{-1}$. Fortunately the divergence is mild and the results on the squeezed configurations, for which $B_{f_{\mathrm{NL}}^{2}}^{n G}$ gives the most important contribution, are insensitive to $k_{\min }$ : changing $k_{\text {min }}=10^{-6} h \mathrm{Mpc}^{-1}$ to $k_{\min }=10^{-9} h \mathrm{Mpc}^{-1}$ results in negligible changes in the squeezed configurations.

On the other hand, the folded and equilateral configurations are more sensitive to $k_{\min }$, and we find that the difference between $k_{\min }=10^{-6} h \mathrm{Mpc}^{-1}$ and $k_{\min }=10^{-9} h \mathrm{Mpc}^{-1}$ is scale-dependent: at $k_{1}=0.01 h \mathrm{Mpc}^{-1}$ the differences are negligible for all shapes, whereas the differences reach $\sim 40 \%$ at $k \sim 1 h \mathrm{Mpc}^{-1}$. (Note that the difference in the squeezed configuration reaches $1 \%$ at $k \sim 1 h \mathrm{Mpc}^{-1}$, being totally negligible on larger scales.) While this divergence does not have much observational consequences (because the signals in the other configurations at $k \gtrsim 0.01 h \mathrm{Mpc}^{-1}$ would be dominated by the other non-linear effects: $B_{m}^{G}$, non-linear bias and terms proportional to $f_{\mathrm{NL}}$, as we show in the next section), there may be a better treatment of the divergence than setting $P_{\phi}(k)=0$ at $k \leqslant k_{\min }$.

\subsection{Scale Dependence}

How important are the primordial non-Gaussianity terms, $B_{f_{\mathrm{NL}}}^{n G 0}, B_{f_{\mathrm{NL}}}^{n G 1}, B_{m}^{n G}, B_{f_{\mathrm{NL}}^{2}}^{n G}$, and $B_{g_{\mathrm{NL}}}^{n G}$, relative to $B_{m}^{G}$ and the non-linear bias term? Which one is the most dominant of the primordial terms, terms proportional to $f_{\mathrm{NL}}, B_{f_{\mathrm{NL}}^{n G}}^{n G}$, or $B_{g_{\mathrm{NL}}}^{n G}$ ? How about the scale-dependence? How about the shape dependence?

We collect all the terms proportional to $f_{\mathrm{NL}}$, and call it $B_{f_{\mathrm{NL}}}^{\text {tot }}$,

$$
\begin{aligned}
B_{f_{\mathrm{NL}}}^{\mathrm{tot}} \equiv & B_{f_{\mathrm{NL}}}^{n G 0}+\frac{\tilde{b}_{2}}{b_{1}}\left[B_{m}^{n G}+B_{f_{\mathrm{NL}}}^{n G 1}\right. \\
& \left.+4\left(\mathcal{G}_{R}\left(k_{1}\right)+\mathcal{G}_{R}\left(k_{2}\right)+\mathcal{G}_{R}\left(k_{3}\right)\right) B_{f_{\mathrm{NL}}}^{n G 0}\right] .
\end{aligned}
$$

Throughout this section, we use the standard value of $\tilde{b}_{2} / b_{1}=$ $3(12 \pi)^{2 / 3} / 20 \simeq 1.68$ from a spherical collapse model.

Figure 10 shows the scale and shape dependence of each term in Equation (51) evaluated at $z=0$. For all configurations shown in this figure, the primordial non-Gaussian term calculated in Sefusatti \& Komatsu (2007) is the smallest among four $f_{\mathrm{NL}}$ terms, which means that the non-Gaussian signal on large scales is much bigger than recognized before. 

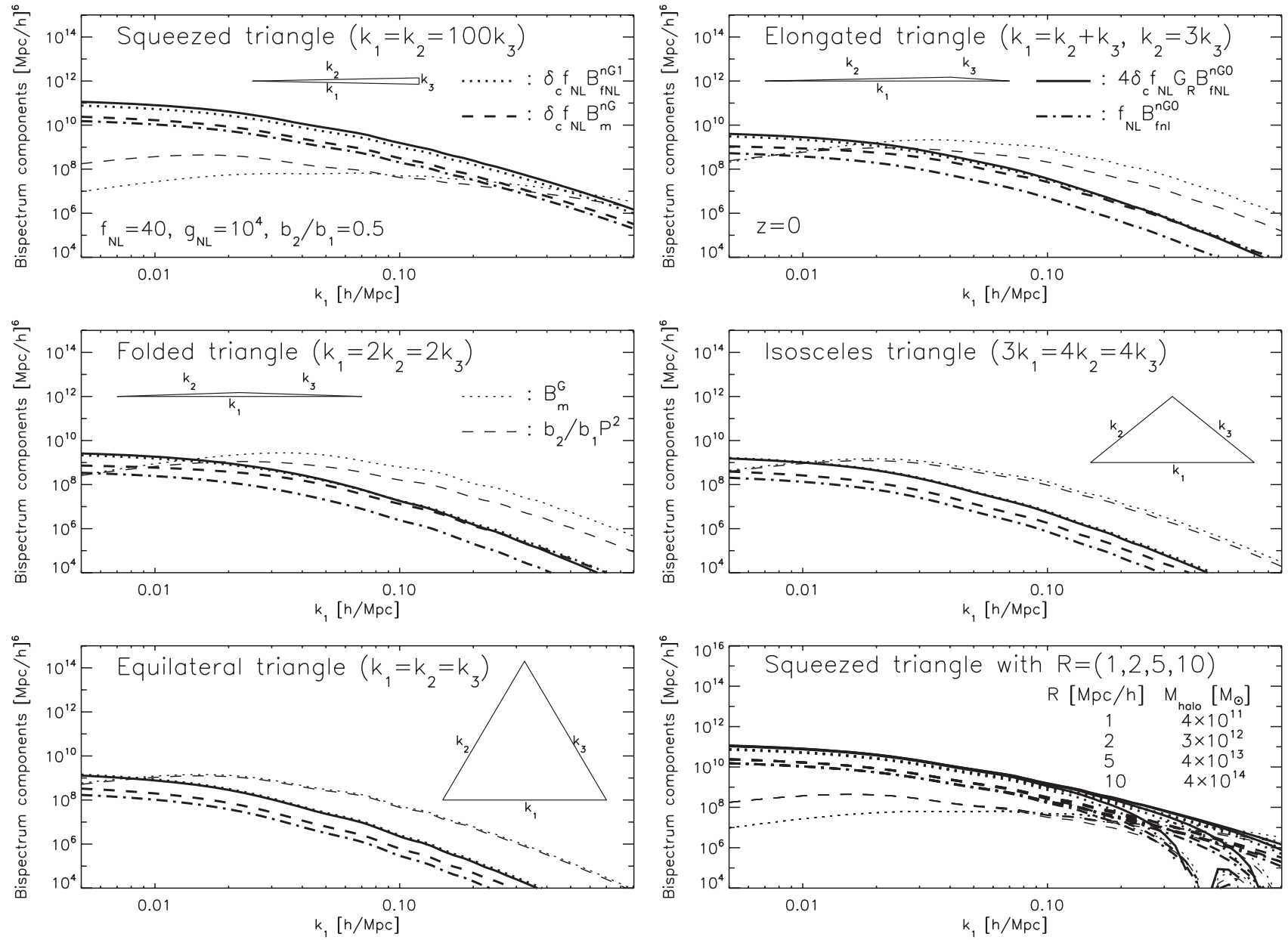

Figure 10. Scale and shape dependence of the galaxy bispectrum terms that are linearly proportional to $f_{\mathrm{NL}}$, as a function of $k_{1}$. Except for the bottom-right panel, we use $R=1 \mathrm{Mpc} h^{-1}$. (Top Left) The squeezed triangles with $k_{1}=k_{2}=100 k_{3}$, (Top Right) the elongated triangles with $k_{1}=k_{2}+k_{3}$ and $k_{2}=3 k_{3}$, (Middle Left) the folded triangles with $k_{1}=2 k_{2}=2 k_{3}$, (Middle Right) the isosceles triangles with $3 k_{1}=4 k_{2}=4 k_{3}$, and (Bottom Left) the equilateral triangles with $k_{1}=k_{2}=k_{3}$. The thick dot-dashed, dashed, solid, and dotted lines show the contributions from the primordial non-Gaussianity: the $B_{f \mathrm{NL}}^{n G 0}($ Equation $(22)), \tilde{b}_{2} / b_{1} B_{m}^{n G}$ (Equation (33)), $4\left(\tilde{b}_{2} / b_{1}\right)\left[\mathcal{G}_{R}\left(k_{1}\right)+\mathcal{G}_{R}\left(k_{2}\right)+\mathcal{G}_{R}\left(k_{3}\right)\right] B_{f_{\mathrm{NL}}}^{n G 0}\left(\mathcal{G}_{R}(k)\right.$ defined in Equation (36)), and $\tilde{b}_{2} / b_{1} B_{f_{\mathrm{NL}}}^{n G 1}$ (Equation (39)) terms, respectively. The thin dotted and dashed lines show the non-linear effects: $B_{m}^{G}$ (Equation (21)) and the non-linear bias (the second term in Equation (9)), respectively. We use the standard value of $\tilde{b}_{2} / b_{1} \equiv \delta_{c} \simeq 1.686$ from spherical collapse model. (Bottom Right) Dependence of the squeezed bispectrum on the smoothing scale, $R$, showing that the dependence is negligible for $k_{1} \ll 1 / R$.

For the squeezed triangle, all of the terms in Equation (51) depend on $k_{1}$ in a similar way. We find their ratios by comparing Equations (44), (45), and (46),

$$
B_{f_{\mathrm{NL}}}^{n G 0}: B_{m}^{n G}: B_{f_{\mathrm{NL}}}^{n G 1} \simeq 1: \frac{26}{28}: 2.96 .
$$

Note that we have used the numerical value of $\mathcal{H}\left(\boldsymbol{k}_{1}, \boldsymbol{k}_{2}\right) \simeq$ 0.741 for $\alpha=100$, and this value slightly increases when $\alpha$ decreases. ${ }^{5}$ Therefore, for the squeezed triangle, we find a simple and illuminating result

$$
B_{f_{\mathrm{NL}}}^{\text {tot }}\left(k_{1}, k_{2}, k_{3}\right) \simeq 15 B_{f_{\mathrm{NL}}}^{n G 0}\left(k_{1}, k_{2}, k_{3}\right) .
$$

This is an important result, showing that the statistical error on $f_{\mathrm{NL}}$ from the galaxy bispectrum will be smaller by at least a factor of 15 , compared to what was obtained in Sefusatti \& Komatsu (2007). Note that this result is valid only for the high-density peak limit, i.e., $v \gg 1$, which implies

\footnotetext{
5 On large scales, $k<0.01 h \mathrm{Mpc}^{-1}$, the numerical ratio $B_{f_{\mathrm{NL}}}^{n G 1} / B_{f_{\mathrm{NL}}}^{n G 0}$ is constant, and is equal to $3.15,3.06,3.00$, and 2.98 for $\alpha=10,20,50$, and 100, respectively.
}

$\tilde{b}_{2} / b_{1}=1.68$. For lower density peaks we would find a smaller factor.

Figure 11 and the top panels of Figure 12 show various bispectrum terms in various triangle configurations (see Figure 1 for the visual representations of the triangles), evaluated at $z=0$. As an example we use the following bias and non-Gaussianity parameters: $b_{2} / b_{1}=0.5, f_{\mathrm{NL}}=40$, and $g_{\mathrm{NL}}=10^{4}$. The value of the linear bias, $b_{1}$, is irrelevant here as it does not change the relative importance of terms in Equation (14), and thus we show the bispectrum terms divided by $b_{1}^{3}$.

The message is quite simple: it is the squeezed configuration that provides the best window into the primordial nonGaussianity. The other non-linear effects become more and more dominant as we move from the squeezed to the equilateral, i.e., panels (a) to (e) in Figure 1. Even with this generous amount of non-Gaussian signals, $f_{\mathrm{NL}}=40$ and $g_{\mathrm{NL}}=10^{4}$, only $f_{\mathrm{NL}}$ term can be visible in the isosceles and equilateral configurations on large scales.

For the the non-squeezed configurations, the $f_{\mathrm{NL}}^{2}$ and $g_{\mathrm{NL}}$ terms with the above chosen parameters are comparable and the $f_{\mathrm{NL}}$ term is order of magnitude greater than the $f_{\mathrm{NL}}^{2}$ and $g_{\mathrm{NL}}$ 

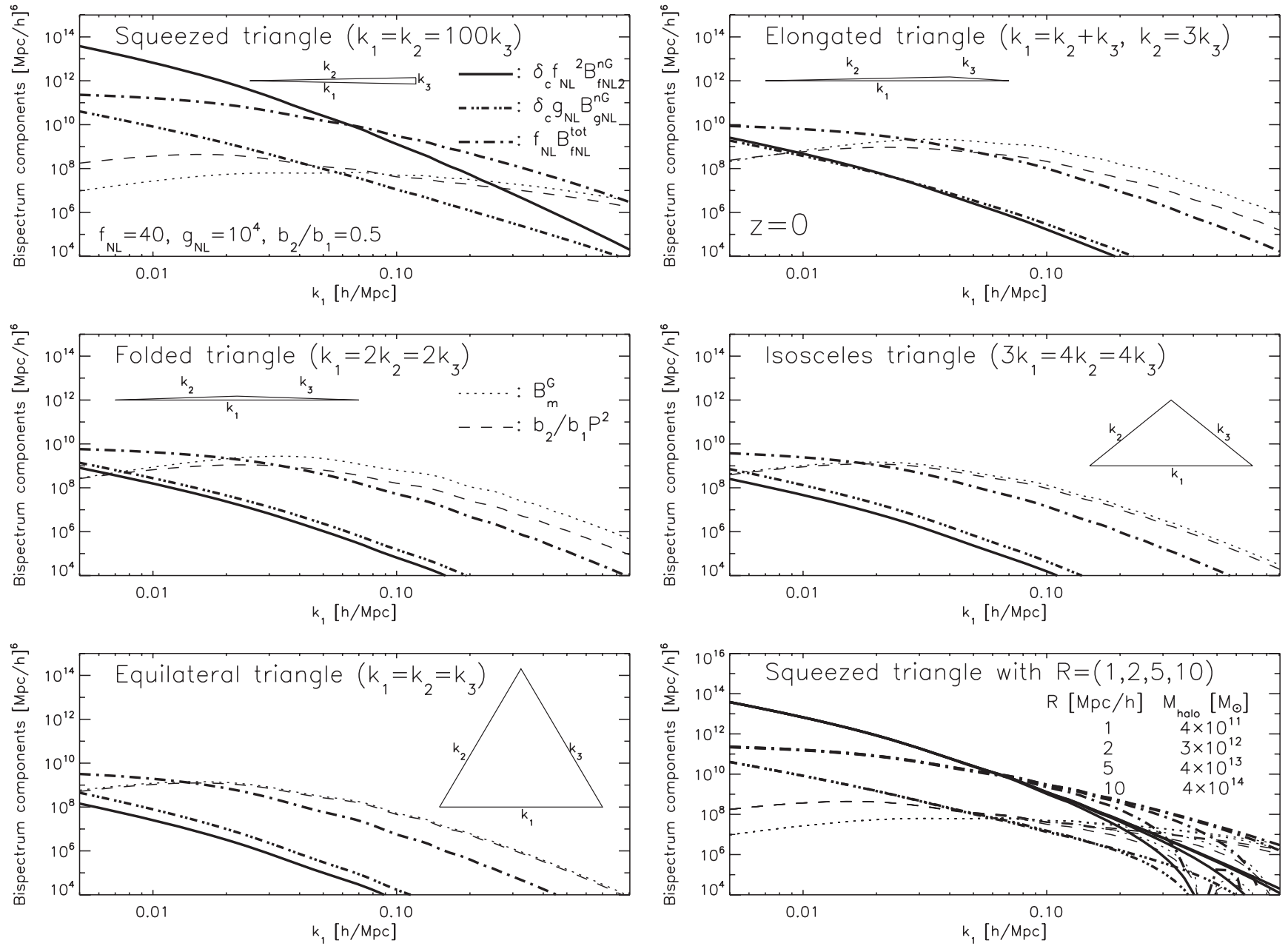

Figure 11. Scale and shape dependence of various bispectrum terms, $B\left(k_{1}, k_{2}, k_{3}\right)$, as a function of $k_{1}$. For the figure except for the bottom right, we use $R=1 \mathrm{Mpc} h^{-1}$. (Top Left) The squeezed triangles with $k_{1}=k_{2}=100 k_{3}$, (Top Right) the elongated triangles with $k_{1}=k_{2}+k_{3}$ and $k_{2}=3 k_{3}$, (Middle Left) the folded triangles with $k_{1}=2 k_{2}=2 k_{3}$, (Middle Right) the isosceles triangles with $3 k_{1}=4 k_{2}=4 k_{3}$, and (Bottom Left) the equilateral triangles with $k_{1}=k_{2}=k_{3}$. The thick dot-dashed, triple-dot-dashed, and solid lines show the contributions from the primordial non-Gaussianity: the $f_{\mathrm{NL}} B_{f_{\mathrm{NL}}}^{\text {tot }}$ (Equation $(51)$ ), $\tilde{b}_{2} / b_{1} g_{\mathrm{NL}} B_{g_{\mathrm{NL}}}^{n G}$ (Equation (30)), and $\tilde{b}_{2} / b_{1} f_{\mathrm{NL}}^{2} B_{f_{\mathrm{NL}}^{2}}^{n G}$ (Equation (31)) terms, respectively. The thin dotted and dashed lines show the non-linear effects: $B_{m}^{G}$ (Equation (21)) and the non-linear bias (the second term in Equation (9)), respectively. We use the standard value of $\tilde{b}_{2} / b_{1} \equiv \delta_{c} \simeq 1.686$ from spherical collapse model. (Bottom Right) Dependence of the squeezed bispectrum on the smoothing scale, $R$, showing that the dependence is negligible for $k_{1} \ll 1 / R$.

terms; however, the $f_{\mathrm{NL}}^{2}$ term is the most dominant of all on large scales in the squeezed configuration $(\alpha>10)$.

We can understand these results analytically by comparing Equations (53), (44), (49), and (50). For the squeezed triangles with $k_{1}=k_{2}=\alpha k_{3}(\alpha \gg 1)$ and a scale-invariant spectrum, $P_{\phi} \propto k^{-3}$, we find

$$
\begin{aligned}
\frac{f_{\mathrm{NL}} B_{f_{\mathrm{NL}}}^{\mathrm{tot}}}{f_{\mathrm{NL}}^{2} B_{f_{\mathrm{NL}}^{2}}^{n G}} & \simeq \frac{15}{f_{\mathrm{NL}} \alpha^{2}} \frac{\mathcal{M}_{0} k^{2}}{4 \mathcal{I}_{0}\left(\tilde{b}_{2} / b_{1}\right)} \\
& \simeq 0.0240\left(\frac{100}{\alpha}\right)^{2} \frac{40}{f_{\mathrm{NL}}}\left(\frac{k}{0.01 h \mathrm{Mpc}^{-1}}\right)^{2},
\end{aligned}
$$

$$
\begin{aligned}
\frac{g_{\mathrm{NL}} B_{g_{\mathrm{NL}}}^{n G}}{f_{\mathrm{NL}}^{2} B_{f_{\mathrm{NL}}^{2}}^{n G}} & \simeq \frac{3}{2 \alpha^{2}} \frac{g_{\mathrm{NL}}}{f_{\mathrm{NL}}^{2}} \\
& \simeq 0.000938\left(\frac{100}{\alpha}\right)^{2}\left(\frac{40}{f_{\mathrm{NL}}}\right)^{2} \frac{g_{\mathrm{NL}}}{10^{4}}
\end{aligned}
$$

$$
\begin{aligned}
\frac{f_{\mathrm{NL}} B_{f_{\mathrm{NL}}}^{\text {tot }}}{g_{\mathrm{NL}} B_{g_{\mathrm{NL}}}^{n G}} & \simeq 15 \frac{f_{\mathrm{NL}}}{g_{\mathrm{NL}}} \frac{\mathcal{M}_{0} k^{2}}{6 \mathcal{I}_{0}} \\
& \simeq 25.6 \frac{f_{\mathrm{NL}}}{40} \frac{10^{4}}{g_{\mathrm{NL}}}\left(\frac{k}{0.01 h \mathrm{Mpc}^{-1}}\right)^{2} .
\end{aligned}
$$

These estimates confirm that $B_{f_{\mathrm{NL}}^{2}}$ dominates over $B_{f_{\mathrm{NL}}}$ and $B_{g_{\mathrm{NL}}}$ in the squeezed configurations on large scales, $k \lesssim$ $0.05 h \mathrm{Mpc}^{-1}$ for $\alpha=100$, and $k \lesssim 0.03 h \mathrm{Mpc}^{-1}$ for $\alpha=50$. For $\alpha=10, f_{\mathrm{NL}}^{2}$ term dominates only on the extremely large scales: $k \lesssim 0.006 h \mathrm{Mpc}^{-1}$.

Note that for a given configuration (for a given $\alpha$ ), $B_{f_{\mathrm{NL}}}^{n G} / B_{f_{\mathrm{NL}}^{2}}^{n G} \propto k^{2}$ and $B_{f_{\mathrm{NL}}}^{n G} / B_{g_{\mathrm{NL}}}^{n G} \propto k^{2}$ while $B_{g_{\mathrm{NL}}}^{n G} / B_{f_{\mathrm{NL}}^{2}}^{n G}$ is independent of $k$, which is consistent with what we show in Figure 11 on $k \lesssim 0.1 h \mathrm{Mpc}^{-1}$.

In summary, the most unexpected and important results of our study are as follows.

1. The terms that are linearly proportional to $f_{\mathrm{NL}}$, derived in Sefusatti \& Komatsu (2007), receive additional 

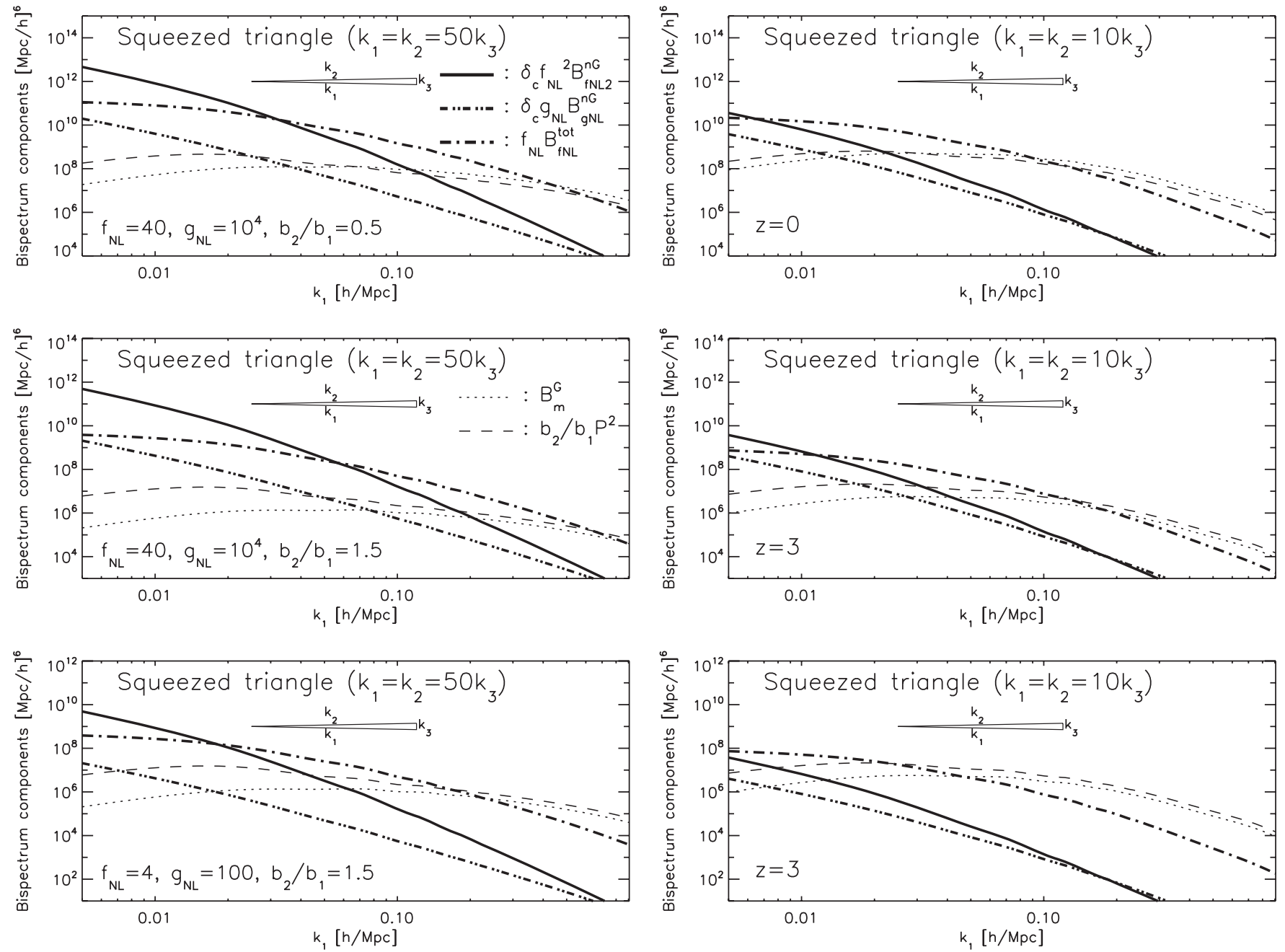

Figure 12. Same as Figure 11, but for squeezed triangles with different ratios: $\alpha=50$ and $\alpha=10$. (Top) All the parameters are the same as in Figure 11. (Middle) $z=3$ and $b_{2} / b_{1}=1.5$. The non-Gaussianity parameters, $f_{\mathrm{NL}}=40$ and $g_{\mathrm{NL}}=10^{4}$, are the same as in Figure 11 . (Bottom) $z=3$ and $b_{2} / b_{1}=1.5$. The non-Gaussianity parameters, $f_{\mathrm{NL}}=4$ and $g_{\mathrm{NL}}=100$.

contributions, and are enhanced by a factor of $\sim 15$ for the squeezed triangles (see Equation (53)).

2. The $f_{\mathrm{NL}}^{2}$ (or $\tau_{\mathrm{NL}}$ ) term actually dominates over the $f_{\mathrm{NL}}$ term by a large factor for the squeezed triangles (see the top-left panel of Figure 11).

This suggests that the galaxy bispectrum is more sensitive to $f_{\mathrm{NL}}$ than previously recognized by Sefusatti \& Komatsu (2007), greatly enhancing our ability to detect the primordial non-Gaussianity of local type. On very large scales, $k_{1} \ll$ $0.01 h \mathrm{Mpc}^{-1}$, even the $g_{\mathrm{NL}}$ term (with $g_{\mathrm{NL}}=10^{4}$ ) dominates over the $f_{\mathrm{NL}}$ term, giving us a hope that perhaps we can obtain a meaningful limit on this term using the galaxy bispectrum.

\subsection{Redshift Dependence}

The quantities we have calculated so far are evaluated at the present epoch, $z=0$. At higher redshift, each quantity needs to be scaled with some powers of the linear growth factor $D(z)$, which is normalized to 1 at the present epoch. We find $P_{R} \propto D^{2}(z), B_{m}^{G} \propto D^{4}(z), B_{f_{\mathrm{NL}}}^{n G 0} \propto D^{3}(z), B_{m}^{n G} \propto D^{3}(z)$, $B_{f_{\mathrm{NL}}}^{n G 1} \propto D^{3}(z), B_{f_{\mathrm{NL}}^{2}}^{n G} \propto D^{2}(z)$, and $B_{g_{\mathrm{NL}}}^{n G} \propto D^{2}(z)$. Therefore, the final result for the halo bispectrum from the local type non-
Gaussianity is

$$
\begin{aligned}
B_{g}\left(k_{1}, k_{2}, k_{3}, z\right)= & b_{1}^{3}(z) D^{4}(z)\left[B_{m}^{G}\left(k_{1}, k_{2}, k_{3}\right)\right. \\
& +\frac{b_{2}(z)}{b_{1}(z)}\left\{P_{R}\left(k_{1}\right) P_{R}\left(k_{2}\right)+(2 \text { cyclic })\right\} \\
& +\frac{f_{\mathrm{NL}}}{D(z)} B_{f_{\mathrm{NL}}}^{n G 0}\left(k_{1}, k_{2}, k_{3}\right) \\
& +\frac{\tilde{b}_{2}(z)}{b_{1}(z)}\left\{\frac { f _ { \mathrm { NL } } } { D ( z ) } \left(B_{m}^{n G}\left(k_{1}, k_{2}, k_{3}\right)+4\left(\mathcal{G}_{R}\left(k_{1}\right)\right.\right.\right. \\
& \left.\left.+\mathcal{G}_{R}\left(k_{2}\right)+\mathcal{G}_{R}\left(k_{3}\right)\right) B_{f_{\mathrm{NL}}}^{n G 0}\left(k_{1}, k_{2}, k_{3}\right)\right) \\
& +B_{f_{\mathrm{NL}}}^{n G 1}\left(k_{1}, k_{2}, k_{3}\right)+\frac{f_{\mathrm{NL}}^{2}}{D^{2}(z)} B_{f_{\mathrm{NL}}^{2}}^{n G}\left(k_{1}, k_{2}, k_{3}\right) \\
& \left.\left.+\frac{g_{\mathrm{NL}}}{D^{2}(z)} B_{g_{\mathrm{NL}}}^{n G}\left(k_{1}, k_{2}, k_{3}\right)\right\}\right],
\end{aligned}
$$

where $B_{m}^{G}, P_{R}, B_{f_{\mathrm{NL}}}^{n G 0}, B_{m}^{n G}, B_{f_{\mathrm{NL}}}^{n G 1}, B_{f_{\mathrm{NL}}^{2}}^{n G}$, and $B_{g_{\mathrm{NL}}}^{n G}$ are evaluated at $z=0$.

From Equation (57) it is clear that the contributions from nonGaussian initial conditions become more and more important as we go to higher redshifts. The new terms that we have derived 

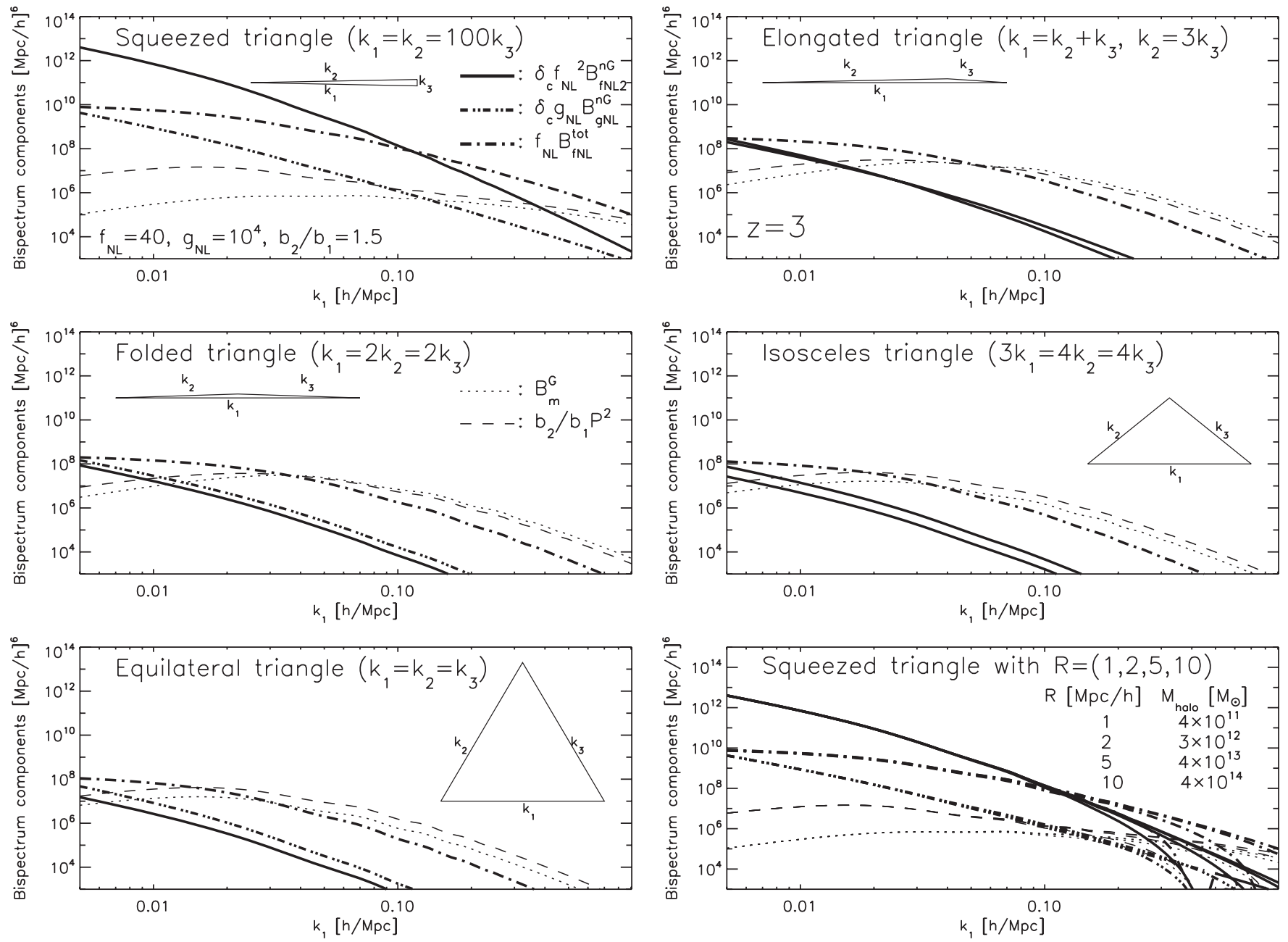

Figure 13. Same as Figure 11, but for $z=3$ and $b_{2} / b_{1}=1.5$. The non-Gaussianity parameters, $f_{\mathrm{NL}}=40$ and $g_{\mathrm{NL}}=10^{4}$, are the same as in Figure 11 .

in this paper, the $B_{f_{\mathrm{N}}^{2}}^{n G}$ and $B_{g_{\mathrm{NL}}}^{n G}$ terms, are even more important than the term derived by Sefusatti \& Komatsu (2007), $B_{f_{\mathrm{NL}}}^{n G 0}$, This property makes high-redshift galaxy surveys particularly a powerful probe of primordial non-Gaussianity.

Figure 13 and the middle panel of Figure 12 show the bispectrum terms at $z=3$. Note that we use a larger value for the non-linear bias, $b_{2} / b_{1}=1.5$, in accordance with a halo model (Cooray \& Sheth 2002). At this redshift, with $f_{\mathrm{NL}}=40$ and $g_{\mathrm{NL}}=10^{4}$, the $g_{\mathrm{NL}}$ and $f_{\mathrm{NL}}^{2}$ terms dominate over the non-linear effects also in the elongated, folded and isosceles configurations at $k \lesssim 0.01 h \mathrm{Mpc}^{-1}$, as well as in the squeezed ones. The $f_{\mathrm{NL}}$ terms dominate over the non-linear effects on even smaller scales, and the importance of the $f_{\mathrm{NL}}^{2}$ and $g_{\mathrm{NL}}$ terms relative to the $f_{\mathrm{NL}}$ term is greater, as expected from their dependence on $D(z)$.

\section{DISCUSSION AND CONCLUSIONS}

Let us come back to this question, "can we still use Sefusatti \& Komatsu's equation, Equation (1), with $b_{1}$ replaced by the scale-dependent bias, Equation (4)?" The answer is clearly no: the primordial non-Gaussianity gives the largest signal in the squeezed limit, whereas the non-linear gravitational evolution and non-linear bias give the minimal signals in the same limit. This means that these effects are physically totally distinct, and thus a mere scale-dependent rescaling of one effect does not give another. Therefore, replacing $b_{1}$ in Equation (1) with the scaledependent bias in Equation (4) results in an incorrect prediction. For example, even though we have a term similar to that of the scale-dependent bias, $B_{m}^{n G}$, in our final expression of the galaxy bispectrum for the local-type primordial non-Gaussianity, there are many more terms that do not look like the scale-dependent bias that appears in the galaxy power spectrum. Furthermore, $B_{m}^{n G}$ is by no means the most dominant term.

In this paper, we have derived a general expression for the bispectrum of density peaks in the presence of primordial non-Gaussianity (Equation (9)), using the MLB formula as well as using the local bias ansatz. This result is general as long as we consider the bispectrum of high density peaks, i.e., $v=\delta_{c} / \sigma_{R} \gg 1$, which is equivalent to highly biased galaxy populations, $b_{1} \gg 1$, on large scales in which the $n$ point correlation functions are much smaller than unity. (This condition was necessary for us to Taylor-expand the exponential in Equation (6).)

We have applied our formula to the local form of primordial non-Gaussianity in Bardeen's curvature perturbations, $\Phi=$ $\phi+f_{\mathrm{NL}} \phi^{2}+g_{\mathrm{NL}} \phi^{3}$, and found new terms that are proportional to $f_{\mathrm{NL}}, f_{\mathrm{NL}}^{2}$ and $g_{\mathrm{NL}}$, which were absent in the formula derived by Sefusatti \& Komatsu (2007). We have examined the shape and scale dependence of these new terms as well as those of the known terms, and found that the primordial non-Gaussianity contributions yield the largest signals in the squeezed triangle 

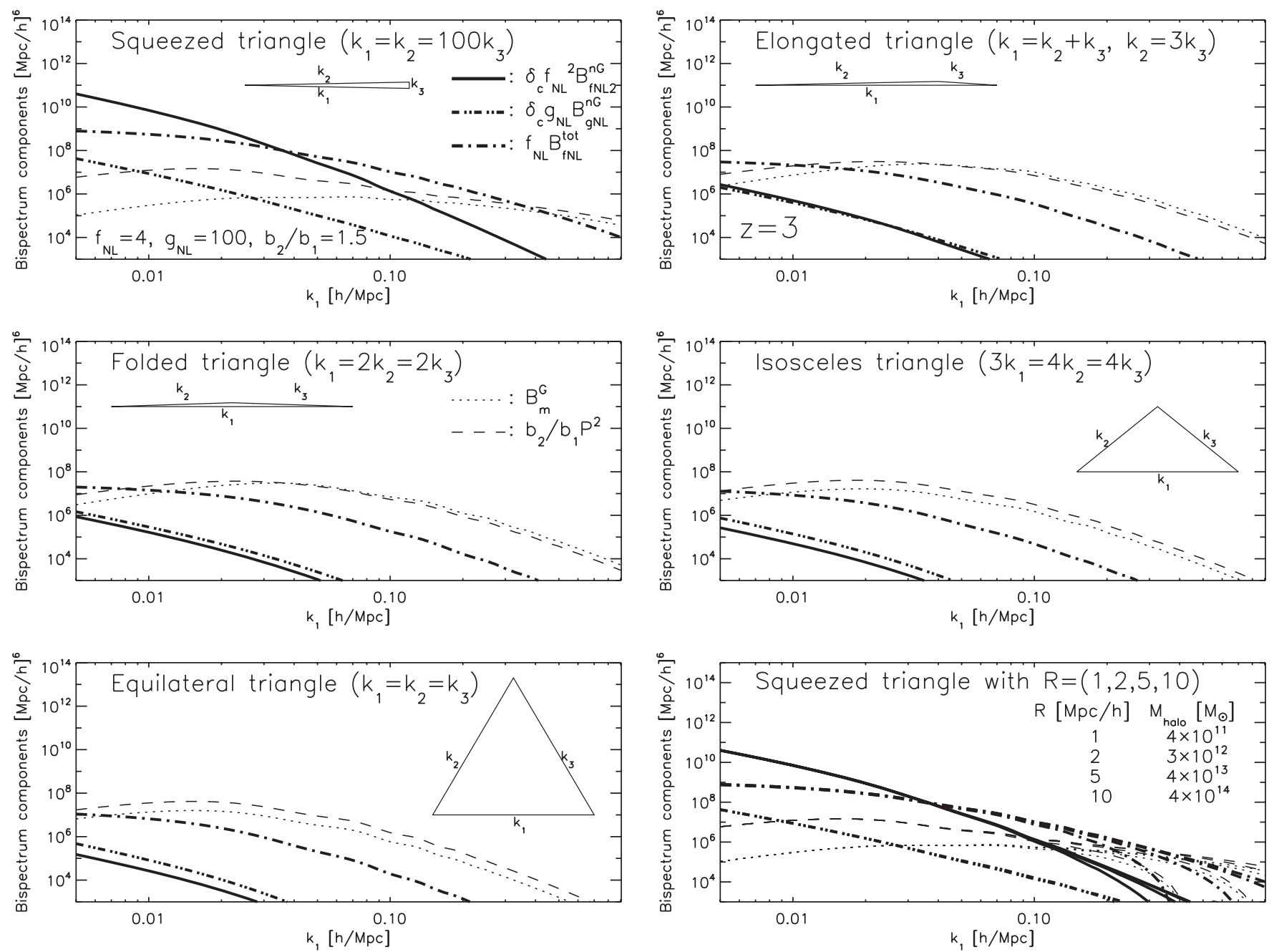

Figure 14. Same as Figure 13, but for smaller non-Gaussianity parameters, $f_{\mathrm{NL}}=4$ and $g_{\mathrm{NL}}=100$.

configurations, where the non-linear gravitational evolution and non-linear bias yield the minimal signals. This is a good news: this property enables us to distinguish the primordial and nonprimordial effects easily.

The effects of primordial non-Gaussianity on the galaxy bispectrum are more important in a high redshift universe, and thus high-redshift galaxy surveys are particularly a potent probe of the physics of inflation via measurements of primordial nonGaussianity.

The most significant conclusion of this paper is that, in the squeezed configurations, the $f_{\mathrm{NL}}^{2}$ term actually dominates over the $f_{\mathrm{NL}}$ term by a large factor, and, on large scales, newly derived $f_{\mathrm{NL}}$ term dominates over the non linear terms for all configurations. Because of this, the galaxy bispectrum should be more sensitive to $f_{\mathrm{NL}}$ than previously recognized: in the high density peak limit, we have found a factor of $\sim 15$ enhancement for the $f_{\mathrm{NL}}$ term studied in Sefusatti \& Komatsu (2007). In addition it is also sensitive to a new term, $g_{\mathrm{NL}}$. Figure 14 and the bottom panel of Figure 12 shows the bispectrum at $z=3$ with much reduced primordial non-Gaussianity parameters, $f_{\mathrm{NL}}=4$ and $g_{\mathrm{NL}}=100$. In the squeezed configurations $(\alpha=100)$, the $f_{\mathrm{NL}}^{2}$ term is still well above the usual terms from Gaussian initial conditions at $k \lesssim 0.1 \mathrm{~h} \mathrm{Mpc}^{-1}$, the $f_{\mathrm{NL}}$ term is above at $k \lesssim 0.4 \mathrm{Mpc}^{-1}$, and the $g_{\mathrm{NL}}$ term is above at $k \lesssim 0.01 h \mathrm{Mpc}^{-1}$. Even with the milder squeezed limit for $\alpha=10$, the $f_{\mathrm{NL}}$ term still is above the Gaussian term at $k \lesssim 0.02 \mathrm{Mpc}^{-1}$.
The fact that the $f_{\mathrm{NL}}^{2}$ term dominates in the squeezed limit is particularly interesting, as it provides us with the unique window into the physics of inflation in the following way. Recently, a number of groups (e.g., Boubekeur \& Lyth 2006; Huang \& Shiu 2006; Byrnes et al. 2006; Buchbinder et al. 2008) have shown that the primordial trispectrum can in general be written as

$$
\begin{aligned}
T_{\Phi}\left(\boldsymbol{k}_{1}, \boldsymbol{k}_{2}, \boldsymbol{k}_{3}, \boldsymbol{k}_{4}\right)= & 6 g_{\mathrm{NL}}\left[P_{\phi}\left(k_{1}\right) P_{\phi}\left(k_{2}\right) P_{\phi}\left(k_{3}\right)+(3 \text { cyclic })\right] \\
& +\frac{25}{18} \tau_{\mathrm{NL}}\left[P_{\phi}\left(k_{1}\right) P_{\phi}\left(k_{2}\right)\left\{P_{\phi}\left(k_{13}\right)+P_{\phi}\left(k_{14}\right)\right\}\right. \\
& +(11 \text { cyclic })],
\end{aligned}
$$

instead of Equation (17). Different models of the early universe predict different relations between $\tau_{\mathrm{NL}}$ and $f_{\mathrm{NL}}$. Therefore, separately detecting the $\tau_{\mathrm{NL}}$ (i.e., $f_{\mathrm{NL}}^{2}$ ) and $f_{\mathrm{NL}}$ terms can be a powerful tool for constraining the model of the early universe.

How well can one constrain these parameters with the current or planned future high-redshift galaxy surveys? As we based our analysis in this paper on the assumption of high density peaks, i.e., $v \gg 1$, the relative importance of the new terms depends on how high the peaks (in which the observed galaxies reside) are. For example, for SDSS-LRG sample $(z=0.315$, $\left.n_{g}=1.36 \times 10^{-4}[\mathrm{~h} / \mathrm{Mpc}]^{3}\right)$, where $\delta_{c} / \sigma_{R} \simeq 1.57$, the halo model (see Sefusatti \& Komatsu 2007, for a detailed method) gives $\tilde{b}_{2} / b_{1} \simeq 0.5$, which is about a third of the value from the high peak limit that we have used in this paper. Therefore, 
we expect that the non-Gaussian signal for the SDSS-LRG bispectrum is smaller by the same factor. Detailed analysis will be presented in a forthcoming paper.

E.K. thanks Kazuya Koyama for useful discussions which led to Section 2.2, and the organizers for the "Focus Week on NonGaussianities in the Sky," where he received useful comments on the draft of this paper. We also thank Emiliano Sefusatti for useful comments. While we were adding Section 2.2 and the additional trispectrum terms, $T_{R}^{1112}$, Sefusatti (2009), which performed similar calculations, had appeared in preprint form. Our alternative derivation given in Section 2.2 and additional calculations on $T_{R}^{1112}$ are independent of, and agree with, those given in Sefusatti (2009). This material is based in part upon work supported by the Texas Advanced Research Program under Grant No. 003658-0005-2006, by NASA grants NNX08AM29G and NNX08AL43G, and by NSF grants AST-0807649 and PHY0758153. E.K. acknowledges support from an Alfred P. Sloan Fellowship. D.J. acknowledges support from a Wendell Gordon Endowed Graduate Fellowship of the University of Texas at Austin.

\section{APPENDIX A}

\section{INTEGRATION OF $T_{R}^{1112}$}

In the standard perturbation theory, the four-point correlator contained in the definition of $T_{R}^{1112}$ (see Equation (26)) is given by $^{6}$

$$
\begin{aligned}
\left\langle\delta^{(1)}\left(\boldsymbol{k}_{1}\right) \delta^{(1)}\left(\boldsymbol{k}_{2}\right) \delta^{(1)}\left(\boldsymbol{k}_{3}\right) \delta^{(2)}\left(\boldsymbol{k}_{4}\right)\right\rangle= & \int \frac{d^{3} q}{(2 \pi)^{3}} F_{2}^{(s)}\left(\boldsymbol{q}, \boldsymbol{k}_{4}-\boldsymbol{q}\right) \\
& \times\left\langle\delta^{(1)}\left(\boldsymbol{k}_{1}\right) \delta^{(1)}\left(\boldsymbol{k}_{2}\right) \delta^{(1)}\left(\boldsymbol{k}_{3}\right) \delta^{(1)}\right. \\
& \left.\times\left(\boldsymbol{k}_{4}-\boldsymbol{q}\right) \delta^{(1)}(\boldsymbol{q})\right\rangle .
\end{aligned}
$$

For non-Gaussian density fields, the leading order of Equation (A1) contains the ensemble average of products of six Gaussian variables, $\phi$, which gives products of three power spectra, $P_{\phi}$. We find

$$
\begin{aligned}
\int \frac{d^{3} q}{(2 \pi)^{3}} & F_{2}^{(s)}\left(\boldsymbol{q}, \boldsymbol{k}_{4}-\boldsymbol{q}\right)\left\langle\delta^{(1)}\left(\boldsymbol{k}_{1}\right) \delta^{(1)}\left(\boldsymbol{k}_{2}\right) \delta^{(1)}\left(\boldsymbol{k}_{3}\right)\right. \\
& \left.\times \delta^{(1)}\left(\boldsymbol{k}_{4}-\boldsymbol{q}\right) \delta^{(1)}(\boldsymbol{q})\right\rangle \\
= & \int \frac{d^{3} q}{(2 \pi)^{3}} F_{2}^{(s)}\left(\boldsymbol{q}, \boldsymbol{k}_{4}-\boldsymbol{q}\right)\left\langle\delta^{(1)}\left(\boldsymbol{k}_{1}\right) \delta^{(1)}\left(\boldsymbol{k}_{2}\right)\right\rangle \\
& \times\left\langle\delta^{(1)}\left(\boldsymbol{k}_{3}\right) \delta^{(1)}\left(\boldsymbol{k}_{4}-\boldsymbol{q}\right) \delta^{(1)}(\boldsymbol{q})\right\rangle+(\operatorname{cyclic} 123) \\
& +2 \int \frac{d^{3} q}{(2 \pi)^{3}} F_{2}^{(s)}\left(\boldsymbol{q}, \boldsymbol{k}_{4}-\boldsymbol{q}\right)\left\langle\delta^{(1)}\left(\boldsymbol{k}_{1}\right) \delta^{(1)}(\boldsymbol{q})\right\rangle \\
& \times\left\langle\delta^{(1)}\left(\boldsymbol{k}_{2}\right) \delta^{(1)}\left(\boldsymbol{k}_{3}\right) \delta^{(1)}\left(\boldsymbol{k}_{4}-\boldsymbol{q}\right)\right\rangle+(\operatorname{cyclic} 123) \\
= & (2 \pi)^{3}\left[2 f_{\mathrm{NL}} P_{m}\left(k_{1}\right) \mathcal{M}\left(k_{3}\right) \int d^{3} q \mathcal{M}(q)\right. \\
& \times \mathcal{M}\left(\left|\boldsymbol{k}_{4}-\boldsymbol{q}\right|\right) P_{\phi}(q)\left\{P_{\phi}\left(\left|\boldsymbol{k}_{4}-\boldsymbol{q}\right|\right)+2 P_{\phi}\left(k_{3}\right)\right\} \\
& \times F_{2}^{(s)}\left(\boldsymbol{q}, \boldsymbol{k}_{4}-\boldsymbol{q}\right) \delta^{D}\left(\boldsymbol{k}_{12}\right)+4 f_{\mathrm{NL}} \mathcal{M}\left(k_{2}\right) \mathcal{M}\left(k_{3}\right) \\
& \times \mathcal{M}\left(k_{14}\right) P_{m}\left(k_{1}\right) F_{2}^{(s)}\left(-\boldsymbol{k}_{1}, \boldsymbol{k}_{14}\right) \\
& \times\left\{P_{\phi}\left(k_{2}\right) P_{\phi}\left(k_{3}\right)+P_{\phi}\left(k_{2}\right) P_{\phi}\left(k_{14}\right)+P_{\phi}\left(k_{3}\right) P_{\phi}\left(k_{14}\right)\right\} \\
& +(\operatorname{cyclic} 123)] \delta^{D}\left(\boldsymbol{k}_{1234}\right) .
\end{aligned}
$$

Therefore, $T_{R}^{1112}$ is given by

$$
\begin{aligned}
T_{R}^{1112}\left(\boldsymbol{k}_{1}, \boldsymbol{k}_{2}, \boldsymbol{k}_{3}, \boldsymbol{k}_{4}\right) & =\tilde{W}_{R}\left(k_{1}\right) \tilde{W}_{R}\left(k_{2}\right) \tilde{W}_{R}\left(k_{3}\right) \tilde{W}_{R}\left(k_{4}\right) \\
& \times\left[2 f_{\mathrm{NL}} P_{m}\left(k_{1}\right) \mathcal{M}\left(k_{3}\right) \int d^{3} q \mathcal{M}(q)\right. \\
& \times \mathcal{M}\left(\left|\boldsymbol{k}_{4}-\boldsymbol{q}\right|\right) P_{\phi}(q)\left\{P_{\phi}\left(\left|\boldsymbol{k}_{4}-\boldsymbol{q}\right|\right)\right. \\
& \left.+2 P_{\phi}\left(k_{3}\right)\right\} F_{2}^{(s)}\left(\boldsymbol{q}, \boldsymbol{k}_{4}-\boldsymbol{q}\right) \delta^{D}\left(\boldsymbol{k}_{12}\right) \\
& +4 f_{\mathrm{NL}} \mathcal{M}\left(k_{2}\right) \mathcal{M}\left(k_{3}\right) \mathcal{M}\left(k_{14}\right) P_{m}\left(k_{1}\right) \\
& \times F_{2}^{(s)}\left(-\boldsymbol{k}_{1}, \boldsymbol{k}_{14}\right)\left\{P_{\phi}\left(k_{2}\right) P_{\phi}\left(k_{3}\right)+P_{\phi}\left(k_{2}\right)\right. \\
& \left.\left.\times P_{\phi}\left(k_{14}\right)+P_{\phi}\left(k_{3}\right) P_{\phi}\left(k_{14}\right)\right\}+(\operatorname{cyclic} 123)\right],
\end{aligned}
$$

where $\mathcal{M}(k) \equiv \mathcal{M}_{R}(k) / \tilde{W}_{R}(k), \boldsymbol{k}_{i j}=\boldsymbol{k}_{i}+\boldsymbol{k}_{j}$, and (cyclic 123) denotes that the cyclic changes among $\left(\boldsymbol{k}_{1}, \boldsymbol{k}_{2}, \boldsymbol{k}_{3}\right)$. We calculate the sum of $\{1112\}$ terms in Equation (27) by integrating Equation (A3)

$$
\begin{aligned}
& \int \frac{d^{3} q}{(2 \pi)^{3}} T_{R}^{(2)}\left(\boldsymbol{q}, \boldsymbol{k}_{1}-\boldsymbol{q}, \boldsymbol{k}_{2}, \boldsymbol{k}_{3}\right)=8 f_{\mathrm{NL}} \tilde{W}_{R}\left(k_{2}\right) \tilde{W}_{R}\left(k_{3}\right) \mathcal{M}\left(k_{1}\right) \\
& \times \mathcal{M}\left(k_{2}\right) \mathcal{M}\left(k_{3}\right)\left\{P_{\phi}\left(k_{2}\right) P_{\phi}\left(k_{3}\right)\right. \\
& \left.+P_{\phi}\left(k_{2}\right) P_{\phi}\left(k_{1}\right)+P_{\phi}\left(k_{3}\right) P_{\phi}\left(k_{1}\right)\right\} \\
& \times \int \frac{d^{3} q}{(2 \pi)^{3}} \tilde{W}_{R}\left(\left|\boldsymbol{k}_{1}-\boldsymbol{q}\right|\right) \tilde{W}_{R}(q) P_{m}(q) F_{2}^{(s)}\left(-\boldsymbol{q}, \boldsymbol{k}_{1}\right) \\
& +4 f_{\mathrm{NL}} \tilde{W}_{R}\left(k_{2}\right) \tilde{W}_{R}\left(k_{3}\right) \mathcal{M}\left(k_{1}\right)\left[P_{m}\left(k_{2}\right) F_{2}^{(s)}\left(\boldsymbol{k}_{2}, \boldsymbol{k}_{1}\right)\right. \\
& \left.+P_{m}\left(k_{3}\right) F_{2}^{(s)}\left(\boldsymbol{k}_{3}, \boldsymbol{k}_{1}\right)\right] \int \frac{d^{3} q}{(2 \pi)^{3}} \tilde{W}_{R}\left(\left|\boldsymbol{k}_{1}-\boldsymbol{q}\right|\right) \\
& \times \tilde{W}_{R}(q) \mathcal{M}(q) \mathcal{M}\left(\left|\boldsymbol{k}_{1}-\boldsymbol{q}\right|\right)\left\{P_{\phi}(q) P_{\phi}\left(\left|\boldsymbol{k}_{1}-\boldsymbol{q}\right|\right)\right. \\
& \left.+2 P_{\phi}(q) P_{\phi}\left(k_{1}\right)\right\}+8 f_{\mathrm{NL}} \tilde{W}_{R}\left(k_{2}\right) \tilde{W}_{R}\left(k_{3}\right) \mathcal{M}\left(k_{3}\right) \\
& \times P_{m}\left(k_{2}\right) \int \frac{d^{3} q}{(2 \pi)^{3}} \tilde{W}_{R}\left(\left|\boldsymbol{k}_{1}-\boldsymbol{q}\right|\right) \tilde{W}_{R}(q) \\
& \times \mathcal{M}\left(\left|\boldsymbol{k}_{1}-\boldsymbol{q}\right|\right) \mathcal{M}\left(\left|\boldsymbol{k}_{2}+\boldsymbol{q}\right|\right) F_{2}^{(s)}\left(-\boldsymbol{k}_{2}, \boldsymbol{k}_{2}+\boldsymbol{q}\right) \\
& \times\left\{P_{\phi}\left(k_{3}\right) P_{\phi}\left(\left|\boldsymbol{k}_{1}-\boldsymbol{q}\right|\right)+P_{\phi}\left(k_{3}\right) P_{\phi}\left(\left|\boldsymbol{k}_{2}+\boldsymbol{q}\right|\right)\right. \\
& \left.+P_{\phi}\left(\left|\boldsymbol{k}_{1}-\boldsymbol{q}\right|\right) P_{\phi}\left(\left|\boldsymbol{k}_{2}+\boldsymbol{q}\right|\right)\right\}+\left(k_{2} \leftrightarrow k_{3}\right) \\
& +8 f_{\mathrm{NL}} \tilde{W}_{R}\left(k_{2}\right) \tilde{W}_{R}\left(k_{3}\right) \mathcal{M}\left(k_{3}\right) \int \frac{d^{3} q}{(2 \pi)^{3}} \\
& \times \tilde{W}_{R}\left(\left|\boldsymbol{k}_{1}-\boldsymbol{q}\right|\right) \tilde{W}_{R}(q) \mathcal{M}\left(\left|\boldsymbol{k}_{1}-\boldsymbol{q}\right|\right) \\
& \times \mathcal{M}\left(\left|\boldsymbol{k}_{2}+\boldsymbol{q}\right|\right) P_{m}(q) F_{2}^{(s)}\left(-\boldsymbol{q}, \boldsymbol{k}_{2}+\boldsymbol{q}\right) \\
& \times\left\{P_{\phi}\left(\left|\boldsymbol{k}_{1}-\boldsymbol{q}\right|\right) P_{\phi}\left(k_{3}\right)+P_{\phi}\left(\left|\boldsymbol{k}_{1}-\boldsymbol{q}\right|\right) P_{\phi}\left(\left|\boldsymbol{k}_{2}+\boldsymbol{q}\right|\right)\right. \\
& \left.+P_{\phi}\left(k_{3}\right) P_{\phi}\left(\left|\boldsymbol{k}_{2}+\boldsymbol{q}\right|\right)\right\}+\left(k_{2} \leftrightarrow k_{3}\right) \\
& +8 f_{\mathrm{NL}}\left(\tilde{W}_{R}\left(k_{2}\right) \tilde{W}_{R}\left(k_{3}\right)\right)^{2} P_{m}\left(k_{3}\right) \mathcal{M}\left(k_{2}\right) \\
& \times \int \frac{d^{3} q}{(2 \pi)^{3}} \mathcal{M}(q) \mathcal{M}\left(\left|\boldsymbol{k}_{2}-\boldsymbol{q}\right|\right) P_{\phi}(q) \\
& \times\left\{P_{\phi}\left(\left|\boldsymbol{k}_{2}-\boldsymbol{q}\right|\right)+2 P_{\phi}\left(k_{2}\right)\right\} F_{2}^{(s)}\left(\boldsymbol{q}, \boldsymbol{k}_{2}-\boldsymbol{q}\right) \\
& +\left(k_{2} \leftrightarrow k_{3}\right) \text {. }
\end{aligned}
$$

\section{APPENDIX B}

\section{SUMMARY OF EQUATIONS}

As various terms contributing to the galaxy bispectrum are scattered over various places in the paper, we collect them together in this appendix. For galaxies of size $R$ (or mass

\footnotetext{
6 Sefusatti (2009) also derived and studied this term independently.
} 
$M=(4 \pi / 3) R^{3} \bar{\rho}_{m}$, where $\bar{\rho}_{m}$ is the cosmic mean matter density), the galaxy bispectrum at redshift $z$ is given by

$$
\begin{aligned}
& B_{g}\left(k_{1}, k_{2}, k_{3}, z\right)=b_{1}^{3}(z) D^{4}(z)\left[B_{m}^{G}\left(k_{1}, k_{2}, k_{3}\right)+\frac{b_{2}(z)}{b_{1}(z)}\right. \\
& \times\left\{P_{R}\left(k_{1}\right) P_{R}\left(k_{2}\right)+(2 \text { cyclic })\right\}+\frac{f_{\mathrm{NL}}}{D(z)} \\
& \times B_{f_{\mathrm{NL}}}^{n G 0}\left(k_{1}, k_{2}, k_{3}\right)+\frac{\tilde{b}_{2}(z)}{b_{1}(z)}\left\{\frac { f _ { \mathrm { NL } } } { D ( z ) } \left(B_{m}^{n G}\left(k_{1}, k_{2}, k_{3}\right)\right.\right. \\
& \left.+4\left(\mathcal{G}_{R}\left(k_{1}\right)+\mathcal{G}_{R}\left(k_{2}\right)+\mathcal{G}_{R}\left(k_{3}\right)\right) B_{f_{\mathrm{NL}}}^{n G 0}\left(k_{1}, k_{2}, k_{3}\right)\right) \\
& +B_{f_{\mathrm{NL}}}^{n G 1}\left(k_{1}, k_{2}, k_{3}\right)+\frac{f_{\mathrm{NL}}^{2}}{D^{2}(z)} B_{f_{\mathrm{NL}}^{2}}^{n G}\left(k_{1}, k_{2}, k_{3}\right) \\
& \left.\left.+\frac{g_{\mathrm{NL}}}{D^{2}(z)} B_{g_{\mathrm{NL}}}^{n G}\left(k_{1}, k_{2}, k_{3}\right)\right\}\right] \text {, }
\end{aligned}
$$

where $b_{1}(z)$ and $b_{2}(z)$ are the linear and non-linear bias parameters, respectively. As we mentioned in Section $2, \tilde{b}_{2}(z) / b_{1}(z)$ would be equal to $\delta_{c}$ within the context of the MLB formalism, but the precise value has to be measured from $N$-body simulations.

Note that the redshift evolution of each term in explicitly given by the powers of the linear growth factor $D(z)$, and various contributions, $B_{m}^{G}, P_{R}, B_{m}^{n G}, B_{f_{\mathrm{NL}}}^{n G 0}, B_{f_{\mathrm{NL}}}^{n G 1}, B_{f_{\mathrm{NL}}^{2}}^{n G}$, and $B_{g_{\mathrm{NL}}}^{n G}$, are evaluated at $z=0$ with

$$
\begin{aligned}
B_{m}^{G}\left(k_{1}, k_{2}, k_{3}\right)= & 2 F_{2}^{(s)}\left(\boldsymbol{k}_{1}, \boldsymbol{k}_{2}\right) \tilde{W}_{R}\left(k_{1}\right) \tilde{W}_{R}\left(k_{2}\right) \tilde{W}_{R}\left(k_{3}\right) \\
& \times P_{m}\left(k_{1}\right) P_{m}\left(k_{2}\right)+(2 \text { cyclic }) \\
B_{f_{\mathrm{NL}}}^{n G 0}\left(k_{1}, k_{2}, k_{3}\right)= & 2 \frac{P_{R}\left(k_{1}\right)}{\mathcal{M}_{R}\left(k_{1}\right)} \frac{P_{R}\left(k_{2}\right)}{\mathcal{M}_{R}\left(k_{2}\right)} \mathcal{M}_{R}\left(k_{3}\right)+(2 \text { cyclic }) \\
B_{m}^{n G}\left(k_{1}, k_{2}, k_{3}\right)= & 4 \tilde{W}_{R}\left(k_{1}\right) \tilde{W}_{R}\left(k_{2}\right) \tilde{W}_{R}\left(k_{3}\right) \\
& \times\left[\frac{\mathcal{F}_{R}\left(k_{1}\right)}{\mathcal{M}_{R}\left(k_{1}\right)}+\frac{\mathcal{F}_{R}\left(k_{2}\right)}{\mathcal{M}_{R}\left(k_{2}\right)}\right] P_{m}\left(k_{1}\right) P_{m}\left(k_{2}\right) \\
& \times F_{2}^{(s)}\left(\boldsymbol{k}_{1}, \boldsymbol{k}_{2}\right)+(2 \text { cyclic }) \\
B_{f_{\mathrm{NL}}}^{n G 1}\left(k_{1}, k_{2}, k_{3}\right) \approx & \frac{1}{2 \sigma_{R}^{2}}\left[8 \tilde{W}_{R}\left(k_{2}\right) \tilde{W}_{R}\left(k_{3}\right) P_{m}\left(k_{2}\right) \mathcal{M}\left(k_{3}\right) P_{\phi}\left(k_{3}\right)\right. \\
& \times \int \frac{d^{3} q}{(2 \pi)^{3}} \tilde{W}_{R}\left(\left|\boldsymbol{k}_{1}-\boldsymbol{q}\right|\right) \tilde{W}_{R}(q) \\
& \times \mathcal{M}\left(\left|\boldsymbol{k}_{1}-\boldsymbol{q}\right|\right) \mathcal{M}\left(\left|\boldsymbol{k}_{2}+\boldsymbol{q}\right|\right) \\
& \times\left[P_{\phi}\left(\left|\boldsymbol{k}_{2}+\boldsymbol{q}\right|\right)+P_{\phi}\left(\left|\boldsymbol{k}_{1}-\boldsymbol{q}\right|\right)\right] \\
& \left.\times F_{2}^{(s)}\left(-\boldsymbol{k}_{2}, \boldsymbol{k}_{2}+\boldsymbol{q}\right)+(5 \text { permutation })\right]
\end{aligned}
$$

$$
\begin{aligned}
B_{f_{\mathrm{NL}}^{2}}^{n G}\left(k_{1}, k_{2}, k_{3}\right) \approx & \frac{1}{2 \sigma_{R}^{2}}\left[8 \mathcal{M}_{R}\left(k_{2}\right) \mathcal{M}_{R}\left(k_{3}\right) P_{\phi}\left(k_{1}\right)\right. \\
& \times\left[P_{\phi}\left(k_{2}\right)+P_{\phi}\left(k_{3}\right)\right] \int \frac{d^{3} q}{(2 \pi)^{3}} \mathcal{M}_{R}(q)
\end{aligned}
$$

Afshordi, N., \& Tolley, A. J. 2008, Phys. Rev. D, 78, 123507

Bartolo, N., Komatsu, E., Matarrese, S., \& Riotto, A. 2004, Phys. Rep., 402, 103

Bernardeau, F., Colombi, S., Gaztañaga, E., \& Scoccimarro, R. 2002, Phys. Rep., 367,1

Boubekeur, L., \& Lyth, D. H. 2006, Phys. Rev. D, 73, 021301

Buchbinder, E. I., Khoury, J., \& Ovrut, B. A. 2008, Phys. Rev. Lett., 100, 171302

Byrnes, C. T., Sasaki, M., \& Wands, D. 2006, Phys. Rev. D, 74, 123519

Cooray, A., \& Sheth, R. 2002, Phys. Rep., 372, 1

Curto, A., Martinez-Gonzalez, E., \& Barreiro, R. B. 2009, arXiv:0902.1523

Dalal, N., Doré, O., Huterer, D., \& Shirokov, A. 2008, Phys. Rev. D, 77, 123514

Desjacques, V., Seljak, U., \& Iliev, I. T. 2008, MNRAS, 396, 85

Fry, J. N., \& Gaztanaga, E. 1993, ApJ, 413, 447

Gangui, A., Lucchin, F., Matarrese, S., \& Mollerach, S. 1994, ApJ, 430, 447

Grinstein, B., \& Wise, M. B. 1986, ApJ, 310, 19

Grossi, M., Verde, L., Carbone, C., Dolag, K., Branchini, E., Iannuzzi, F., Matarrese, S., \& Moscardini, L. 2009, MNRAS, 398, 321

Huang, M.-X., \& Shiu, G. 2006, Phys. Rev. D, 74, 121301

Jeong, D., \& Komatsu, E. 2009, ApJ, 691, 569

Kogo, N., \& Komatsu, E. 2006, Phys. Rev., D73, 083007

Komatsu, E. 2001, PhD thesis, Tohoku Univ. (arXiv:astro-ph/0206039)

Komatsu, E., \& Spergel, D. N. 2001, Phys. Rev. D, 63, 63002

Komatsu, E., et al. 2009, ApJS, 180, 330

Matarrese, S., Lucchin, F., \& Bonometto, S. A. 1986, ApJ, 310, L21

Matarrese, S., \& Verde, L. 2008, ApJ, 677, L77

McDonald, P. 2008, Phys. Rev. D, 78, 123519 
Mo, H. J., \& White, S. D. M. 1996, MNRAS, 282, 347

Mo, H. J., Jing, Y. P., \& White, S. D. M. 1997, MNRAS, 284, 189

Okamoto, T., \& Hu, W. 2002, Phys. Rev. D, 66, 063008

Peebles, P. J. E. 1980, The Large-scale Structure of The Universe (Research supported by the National Science Foundation; Princeton, NJ: Princeton Univ.)

Pillepich, A., Porciani, C., \& Hahn, O. 2008, arXiv:0811.4176

Politzer, H. D., \& Wise, M. B. 1984, ApJ, 285, L1

Salopek, D. S., \& Bond, J. R. 1990, Phys. Rev. D, 42, 3936
Scoccimarro, R., Sefusatti, E., \& Zaldarriaga, M. 2004, Phys. Rev. D, 69, 103513 Sefusatti, E. 2009, arXiv:0905.0717

Sefusatti, E., \& Komatsu, E. 2007, Phys. Rev. D, 76, 083004

Slosar, A., Hirata, C., Seljak, U., Ho, S., \& Padmanabhan, N. 2008, J. Cosmol. Part. Phys., JCAP08(2008)08

Smith, K. M., Senatore, L., \& Zaldarriaga, M. 2009, arXiv:0901.2572

Taruya, A., Koyama, K., \& Matsubara, T. 2008, Phys. Rev. D, 78, 123534

Verde, L., Wang, L., Heavens, A. F., \& Kamionkowski, M. 2000, MNRAS, 313 , 141 